\title{
INCOMPRESSIBLE, INVISCID LIMIT OF THE COMPRESSIBLE NAVIER-STOKES SYSTEM
}

\author{
Nader MASMOUDI ${ }^{1}$ \\ Ceremade, URA CNRS 749, Université Paris IX Dauphine, Place du Maréchal de Lattre de Tassigny, \\ 75775 Paris Cedex 16, France
}

Received in 2 February 2000

ABSTRACT. - We prove some asymptotic results concerning global (weak) solutions of compressible isentropic Navier-Stokes equations. More precisely, we establish the convergence towards solutions of incompressible Euler equations, as the density becomes constant, the Mach number goes to 0 and the Reynolds number goes to infinity.

(C) 2001 L'Association Publications de l'Institut Henri Poincaré. Published by Elsevier B.V. All rights reserved

RÉSUMÉ. - Nous prouvons quelques résultats asymptotiques concernant des solutions (faibles) globales des équations de Navier-Stokes (isentropique) compressible. Plus précisément, nous établissons la convergence vers une solution des équations d'Euler incompressible, lorsque la densité devient constante, le nombre de Mach tend vers 0 et le nombre de Reynolds tend vers l'infini.

(C) 2001 L'Association Publications de l'Institut Henri Poincaré. Published by Elsevier B.V. All rights reserved

\section{Introduction}

From a physical point of view, one can formally derive incompressible models from compressible ones, when the Mach number goes to zero and the density becomes almost constant. In Lions and the author [12], this problem is investigated starting form the global solutions of the compressible Navier-Stokes equations constructed by Lions [11]. We have shown the convergence towards the incompressible Navier-Stokes equations as well as the convergence towards the incompressible Euler equations (if the viscosity coefficients go to zero and if the initial data are "well prepared"). These results have been precised and extended in different works (see [13,2,1,14]).

In this paper, we extend the result shown in [12] concerning the convergence to the Euler system to the case of more general initial data. In fact if the viscosity goes to zero too, we loose spatial compactness properties. In order to circumvent this difficulty, we use energy arguments. Hence, we have to describe (precisely) the oscillations that take place and include them in the energy estimates. Ideas of this type were introduced by

E-mail address: masmoudi@cims.nyu.edu (N. Masmoudi).

${ }^{1}$ Current adress: Courant Institute, New York University 251 Mercer St, New York, NY 10012, USA. 
Schochet [17], (see also [4]) and extended to the case of vanishing viscosity coefficients in [16]. Let us now precise the scalings, we are going to use, which are the same as those used in [12]. The unknowns $(\tilde{\rho}, v)$ are respectively the density and the velocity of the fluid (gaz). We scale $\tilde{\rho}$ and $v$ (and thus $p$ ) in the following way

$$
\tilde{\rho}=\rho(x, \varepsilon t), \quad v=\varepsilon u(x, \varepsilon t),
$$

and we assume that the viscosity coefficients $\tilde{\mu}, \tilde{\xi}(\tilde{\xi}-\tilde{\mu}, \tilde{\mu}$ are called the volumic and dynamical viscosity coefficients) are also small and scale like

$$
\tilde{\mu}=\varepsilon \mu_{\varepsilon}, \quad \tilde{\xi}=\varepsilon \xi_{\varepsilon}
$$

where $\varepsilon \in(0,1)$ is a "small parameter" and the normalized coefficient $\mu_{\varepsilon}, \xi_{\varepsilon}$ satisfy $\mu_{\varepsilon}>0, \xi_{\varepsilon}+\mu_{\varepsilon}>0$. Moreover, we assume that

$$
\mu_{\varepsilon} \rightarrow 0 \quad \text { as } \varepsilon \text { goes to } 0_{+} .
$$

With the preceding scalings, the compressible Navier-Stokes system reads

$$
\left\{\begin{array}{l}
\frac{\partial \rho}{\partial t}+\operatorname{div}(\rho u)=0, \quad \rho \geqslant 0, \\
\frac{\partial \rho u}{\partial t}+\operatorname{div}(\rho u \otimes u)-\mu_{\varepsilon} \Delta u-\xi_{\varepsilon} \nabla \operatorname{div} u+\frac{a}{\varepsilon^{2}} \nabla \rho^{\gamma}=0 .
\end{array}\right.
$$

One can always assume that $a=1 / \gamma$ by replacing $\varepsilon$ by $\sqrt{a \gamma} \varepsilon$. All throughout this paper the domain $\Omega$ will be the the whole space $\mathbb{R}^{N}$ or the torus $\mathbb{T}^{N}$ (in this last case, all the functions are defined on $\mathbb{R}^{N}$ and assumed to be periodic with period $2 \pi a_{i}$ in the $i$ th variable). We recall here that the inviscid limit, namely the convergence of the NavierStokes equations to Euler equations in the case of a domain with boundary is an open problem even in the incompressible case, which seems to be easier (see [15] for a partial result).

\subsection{Statement of the results}

\subsubsection{The whole space case}

Let us begin with the case of the whole space. We consider a sequence of global weak solutions $\left(\rho_{\varepsilon}, u_{\varepsilon}\right)$ of the compressible Navier-Stokes equations (4) and we assume that $\rho_{\varepsilon}-1 \in L^{\infty}\left(0, \infty ; L_{2}^{\gamma}\right) \cap C\left([0, \infty), L_{2}^{p}\right)$ for all $1 \leqslant p<\gamma$, where $L_{2}^{p}=\left\{f \in L_{\text {loc }}^{1}\right.$, $\left.|f| 1_{|f| \geqslant 1} \in L^{p},|f| 1_{|f| \leqslant 1} \in L^{2}\right\}, u_{\varepsilon} \in L^{2}\left(0, T ; H^{1}\right)$ for all $T \in(0, \infty)$ (with a norm which can explode when $\varepsilon$ goes to 0$), \rho_{\varepsilon}\left|u_{\varepsilon}\right|^{2} \in L^{\infty}\left(0, \infty ; L^{1}\right)$ and $\rho_{\varepsilon} u_{\varepsilon} \in C([0, \infty)$; $\left.L^{2 \gamma /(\gamma+1)}-w\right)$ i.e. is continuous with respect to $t \geqslant 0$ with values in $L^{2 \gamma /(\gamma+1)}$ endowed with its weak topology. We require (4) to hold in the sense of distributions and we impose the following conditions at infinity

$$
\rho_{\varepsilon} \rightarrow 1 \quad \text { as }|x| \rightarrow+\infty, \quad u_{\varepsilon} \rightarrow 0 \quad \text { as }|x| \rightarrow+\infty .
$$


Finally, we prescribe initial conditions

$$
\left.\rho_{\varepsilon}\right|_{t=0}=\rho_{\varepsilon}^{0},\left.\quad \rho_{\varepsilon} u_{\varepsilon}\right|_{t=0}=m_{\varepsilon}^{0},
$$

where $\rho_{\varepsilon}^{0} \geqslant 0, \rho_{\varepsilon}^{0}-1 \in L^{\gamma}, m_{\varepsilon}^{0} \in L^{2 \gamma /(\gamma+1)}, m_{\varepsilon}^{0}=0$ a.e. on $\left\{\rho_{\varepsilon}^{0}=0\right\}$ and $\rho_{\varepsilon}^{0}\left|u_{\varepsilon}^{0}\right|^{2} \in L^{1}$, denoting by $u_{\varepsilon}^{0}=m_{\varepsilon}^{0} / \rho_{\varepsilon}^{0}$ on $\left\{\rho_{\varepsilon}^{0}>0\right\}, u_{\varepsilon}^{0}=0$ on $\left\{\rho_{\varepsilon}^{0}=0\right\}$. The initial conditions also satisfy the following uniform bounds

$$
\int \rho_{\varepsilon}^{0}\left|u_{\varepsilon}^{0}\right|^{2}+\frac{1}{\varepsilon^{2}} \int\left(\rho_{\varepsilon}^{0}\right)^{\gamma}-1-\gamma\left(\rho_{\varepsilon}^{0}-1\right) \leqslant C,
$$

where, here and below, $C$ denotes various positive constants independent of $\varepsilon$. Let us notice that (7) implies in particular that, roughly speaking, $\rho_{\varepsilon}^{0}$ is of order $1+\mathrm{O}(\varepsilon)$. In the sequel, we will use the following notation $\rho_{\varepsilon}=1+\varepsilon \varphi_{\varepsilon}$. Notice that if $\gamma<2$, we cannot deduce any bound for $\varphi_{\varepsilon}$ in $L^{\infty}\left(0, T ; L^{2}\right)$. This is why we prefer to work with the following approximation

$$
\Phi_{\varepsilon}=\frac{1}{\varepsilon} \sqrt{\frac{2 a}{\gamma-1}\left(\rho_{\varepsilon}^{\gamma}-1-\gamma\left(\rho_{\varepsilon}-1\right)\right)} .
$$

Furthermore, we assume that $\sqrt{\rho_{\varepsilon}^{0}} u_{\varepsilon}^{0}$ converges strongly in $L^{2}$ to some $\widetilde{u}^{0}$. Then, we denote by $u^{0}=P \tilde{u}^{0}$, where $P$ is the projection on divergence-free vector fields, we also define $Q$ (the projection on gradient vector fields), hence $\tilde{u}^{0}=P \tilde{u}^{0}+Q \tilde{u}^{0}$. Moreover, we assume that $\Phi_{\varepsilon}^{0}$ converges strongly in $L^{2}$ to some $\varphi^{0}$. This also implies that $\varphi_{\varepsilon}^{0}$ converges to $\varphi^{0}$ in $L_{2}^{\gamma}$.

Our last requirement on $\left(\rho_{\varepsilon}, u_{\varepsilon}\right)$ concerns the total energy: we assume that we have

$$
\begin{aligned}
& E_{\varepsilon}(t)+\int_{0}^{t} D_{\varepsilon}(s) \mathrm{d} s \leqslant E_{\varepsilon}^{0} \quad \text { a.e. } t \\
& \frac{d E_{\varepsilon}}{d t}+D_{\varepsilon} \leqslant 0 \quad \text { in } \mathcal{D}^{\prime}(0, \infty)
\end{aligned}
$$

where

$$
\begin{gathered}
E_{\varepsilon}(t)=\int_{\Omega} \frac{1}{2} \rho_{\varepsilon}\left|u_{\varepsilon}\right|^{2}(t)+\frac{a}{\varepsilon^{2}(\gamma-1)}\left(\left(\rho_{\varepsilon}\right)^{\gamma}-1-\gamma\left(\rho_{\varepsilon}-1\right)\right)(t), \\
D_{\varepsilon}(t)=\int_{\Omega} \mu_{\varepsilon}\left|D u_{\varepsilon}\right|^{2}(t)+\xi_{\varepsilon}\left(\operatorname{div} u_{\varepsilon}\right)^{2}(t)
\end{gathered}
$$

and

$$
E_{\varepsilon}^{0}=\int_{\Omega} \frac{1}{2} \rho_{\varepsilon}^{0}\left|u_{\varepsilon}^{0}\right|^{2}+\frac{a}{\varepsilon^{2}(\gamma-1)}\left(\left(\rho_{\varepsilon}^{0}\right)^{\gamma}-1-\gamma\left(\rho_{\varepsilon}^{0}-1\right)\right) .
$$

We now wish to emphasize the fact that we assume the existence of a sequence of solutions with the above properties, and we shall also assume that $\gamma>N / 2$. We recall 
the results of Lions [11] which yield the existence of such solutions precisely when $\gamma>N / 2$ if $N \geqslant 4, \gamma \geqslant 9 / 5$ if $N=3$ and $\gamma \geqslant 3 / 2$ if $N=2$. We also refer to [12] for the proof of the uniform bounds.

When $\varepsilon$ goes to zero and $\mu_{\varepsilon}$ goes to 0 , we expect that $u_{\varepsilon}$ converges to $v$, the solution of the Euler system

$$
\left\{\begin{array}{l}
\partial_{t} v+\operatorname{div}(v \otimes v)+\nabla \pi=0 \\
\operatorname{div} v=\left.0 \quad v\right|_{t=0}=u^{0}
\end{array}\right.
$$

in $C\left(\left[0, T^{*}\right) ; H^{s}\right)$. We show the following theorem

THEOREM 1.1 (The whole space case). - We assume that $\mu_{\varepsilon} \rightarrow{ }_{\varepsilon} 0$ (such that $\mu_{\varepsilon}+$ $\xi_{\varepsilon}>0$ for all $\left.\varepsilon\right)$ and that $P \tilde{u}^{0} \in H^{s}$ for some $s>N / 2+1$, then $P\left(\sqrt{\rho_{\varepsilon}} u_{\varepsilon}\right)$ converges to $v$ in $L^{\infty}\left(0, T ; L^{2}\right)$ for all $T<T^{*}$, where $v$ is the unique solution of the Euler system in $L_{\mathrm{loc}}^{\infty}\left(\left[0, T^{*}\right) ; H^{s}\right)$ and $T^{*}$ is the existence time of $(9)$. In addition $\sqrt{\rho_{\varepsilon}} u_{\varepsilon}$ converges to $v$ in $L^{p}\left(0, T ; L_{\mathrm{loc}}^{2}\right)$ for all $1 \leqslant p<+\infty$ and all $T<T^{*}$.

\subsubsection{The periodic case}

Now, we take $\Omega=\mathbb{T}^{N}$ and consider a sequence of solutions ( $\rho_{\varepsilon}, u_{\varepsilon}$ ) of (4), satisfying the same conditions as in the whole space case (the functions are now periodic in space and all the integration are performed over $\mathbb{T}^{N}$ ). Of course, the conditions at infinity are removed and the spaces $L_{2}^{p}$ can be replaced by $L^{p}$. Here, we have to impose more conditions on the oscillating part (acoustic waves), namely we have to assume that $Q \tilde{u}^{0}$ is more regular than $L^{2}$. In fact, in the periodic case, we do not have a dispersion phenomenon as in the case of the whole space and the acoustic waves will not go to infinity, but they are going to interact with each other. This is way, we have to include them in the energy estimates to show our convergence result.

For the next theorem, we assume that $Q \tilde{u}^{0}, \varphi^{0} \in H^{s-1}$ and that there exists a nonnegative constant $v$ such that $\mu_{\varepsilon}+\xi_{\varepsilon} \geqslant 2 v>0$ for all $\varepsilon$. For simplicity, we assume that $\mu_{\varepsilon}+\xi_{\varepsilon}$ converges to $2 v$.

THEOREM 1.2 (The periodic case). - We assume that $\mu_{\varepsilon} \rightarrow_{\varepsilon} 0$ (such that $\mu_{\varepsilon}+\xi_{\varepsilon} \rightarrow$ $2 v>0)$ and that $P \tilde{u}^{0} \in H^{s}$ for some $s>N / 2+1$, and $Q \tilde{u}^{0}, \varphi^{0} \in H^{s-1}$ then $P\left(\sqrt{\rho_{\varepsilon}} u_{\varepsilon}\right)$ converges to $v$ in $L^{\infty}\left(0, T ; L^{2}\right)$ for all $T<T^{*}$, where $v$ is the unique solution of the Euler system in $L_{\mathrm{loc}}^{\infty}\left(0, T^{*} ; H^{s}\right)$ and $T^{*}$ is the existence time of (9). In addition $\sqrt{\rho_{\varepsilon}} u_{\varepsilon}$ converges weakly to $v$ in $L^{\infty}\left(0, T ; L^{2}\right)$.

In the above theorem, one can remove the condition $2 v>0$. In that case, we still have the result of Theorem 1.2 but only on an interval of time $\left(0, T^{* *}\right)$ which is the existence interval for the equation governing the oscillating part, which will be given later on.

THEOREM 1.3 (The periodic case, $v=0$ ). - We assume that $\mu_{\varepsilon} \rightarrow_{\varepsilon} 0$ (such that $\left.\mu_{\varepsilon}+\xi_{\varepsilon} \rightarrow 0\right)$ and that $\tilde{u}^{0} \in H^{s}$ for some $s>N / 2+1$, and $\varphi^{0} \in H^{s}$ then $P\left(\sqrt{\rho_{\varepsilon}} u_{\varepsilon}\right)$ converges to $v$ in $L^{\infty}\left(0, T ; L^{2}\right)$ for all $T<\inf \left(T^{*}, T^{* *}\right)$, where $v$ is the unique solution of the Euler system in $L_{\mathrm{loc}}^{\infty}\left(0, T^{*} ; H^{s}\right), T^{*}$ is the existence time of (9) and $T^{* *}$ the existence time of (32), with $v=0$. In addition $\sqrt{\rho_{\varepsilon}} u_{\varepsilon}$ converges weakly to $v$ in $L^{\infty}\left(0, T ; L^{2}\right)$. 


\section{The whole space case}

We recall that in the case of the whole space, we do not assume any extra condition on the viscosity $\xi_{\varepsilon}$, neither do we assume any regularity (more than $L^{2}$ ) for the gradient part of the initial data. The proof relies on the dispersion property of the wave equation $[18,1]$ and the notion of non dissipative solutions for the Euler system [10,12].

First, using the energy bounds, we deduce that $\rho_{\varepsilon}-1$ converges to 0 in $L^{\infty}\left(0, T ; L_{2}^{\gamma}\right)$ and that there exists some $u \in L^{\infty}\left(0, T ; L^{2}\right)$ and a subsequence $\sqrt{\rho_{\varepsilon}} u_{\varepsilon}$ converging weakly to $u$. Hence, we also deduce that $\rho_{\varepsilon} u_{\varepsilon}$ converges weakly to $u$ in $L^{2 \gamma /(\gamma+1)}$. We next introduce the following group $(\mathcal{L}(\tau), \tau \in \mathbb{R})$ defined by $\mathrm{e}^{\tau L}$ where $L$ is the operator defined on $\mathcal{D}^{\prime} \times\left(\mathcal{D}^{\prime}\right)^{N}$, by

$$
L\left(\begin{array}{l}
\varphi \\
v
\end{array}\right)=-\left(\begin{array}{c}
\operatorname{div} v \\
\nabla \varphi
\end{array}\right)
$$

It is easy to check that $\mathrm{e}^{\tau L}$ is an isometry on each $H^{s} \times\left(H^{s}\right)^{N}$ for all $s \in \mathbb{R}$ and for all $\tau$. This will allow

$$
\mathrm{e}^{\tau L}\left(\begin{array}{l}
\varphi \\
v
\end{array}\right)=\left(\begin{array}{l}
\varphi(\tau) \\
v(\tau)
\end{array}\right)
$$

solves

$$
\frac{\partial \varphi}{\partial \tau}=-\operatorname{div} v, \quad \frac{\partial v}{\partial \tau}=-\nabla \varphi
$$

and thus $\frac{\partial^{2} \varphi}{\partial \tau^{2}}-\Delta \varphi=0$.

Let $\left(\psi_{\varepsilon}, m_{\varepsilon}=\nabla q_{\varepsilon}\right)$ be the solution of the following system

$$
\begin{cases}\frac{\partial \psi_{\varepsilon}}{\partial t}=-\frac{1}{\varepsilon} \operatorname{div} m_{\varepsilon}, & \psi_{\varepsilon}(t=0)=\Phi_{\varepsilon}^{0}, \\ \frac{\partial m_{\varepsilon}}{\partial t}=-\frac{1}{\varepsilon} \nabla \psi_{\varepsilon}, & m_{\varepsilon}(t=0)=Q \sqrt{\rho_{\varepsilon}^{0}} u_{\varepsilon}^{0} .\end{cases}
$$

We recall that for all $v \in H^{s}$, we define $Q v=\nabla \Delta^{-1} \operatorname{div} v$ and $P v=v-Q v$. We also introduce the following regularizations $\psi_{\varepsilon, \delta}=\psi_{\varepsilon} * \chi_{\delta}, \nabla q_{\varepsilon, \delta}=\nabla q_{\varepsilon} * \chi_{\delta}$, where $\chi \in C_{0}^{\infty}\left(\mathbb{R}^{N}\right)$ such that $\int \chi=1$ and $\chi_{\delta}(x)=\left(1 / \delta^{N}\right) \chi(x / \delta)$. Since (11) is linear it is easy to see that $\left(\psi_{\varepsilon, \delta}, \nabla q_{\varepsilon, \delta}\right)$ is a solution of the same system with regularized initial data. Using (as in [1]) Strichartz type inequality, we get

$$
\left\|\left(\begin{array}{c}
\psi_{\varepsilon, \delta} \\
\nabla q_{\varepsilon, \delta}
\end{array}\right)\right\|_{\left.L^{p}\left(\mathbb{R} ; W^{s, q}\left(\mathbb{R}^{N}\right)\right)\right)} \leqslant C \varepsilon^{1 / p}\left\|\left(\begin{array}{c}
\Phi_{\varepsilon}^{0} \\
Q\left(\sqrt{\rho_{\varepsilon}^{0}} u_{\varepsilon}^{0}\right)
\end{array}\right) * \chi_{\delta}\right\|_{H^{s+\sigma}}
$$

for all $p, q>2$ and $\sigma>0$ such that

$$
\frac{2}{q}=(N-1)\left(\frac{1}{2}-\frac{1}{p}\right), \quad \sigma=\frac{N+1}{N-1} .
$$


This yields that for all fixed $\delta$ and for all $s \in \mathbb{R}$, we have as $\varepsilon$ goes to 0

$$
\left(\psi_{\varepsilon, \delta}, \nabla q_{\varepsilon, \delta}\right) \rightarrow 0 \quad \text { in } L^{p}\left(\mathbb{R} ; W^{s, q}\left(\mathbb{R}^{N}\right)\right) .
$$

Now, we turn to the energy estimates. Let us rewrite the energy inequality for almost all $t$

$$
\frac{1}{2} \int_{\Omega} \rho_{\varepsilon}\left|u_{\varepsilon}\right|^{2}(t)+\Phi_{\varepsilon}^{2}(t)+\int_{0}^{t} \int_{\Omega} \mu_{\varepsilon}\left|D u_{\varepsilon}\right|^{2}+\xi_{\varepsilon}\left(\operatorname{div} u_{\varepsilon}\right)^{2} \leqslant \frac{1}{2} \int_{\Omega} \rho_{\varepsilon}^{0}\left|u_{\varepsilon}^{0}\right|^{2}+\Phi_{\varepsilon}^{2}(0) .
$$

Then, the conservation of energy for $v$ reads

$$
\int_{\Omega} \frac{1}{2}|v|^{2}(t)=\int_{\Omega} \frac{1}{2}\left|u_{0}\right|^{2}
$$

and using the fact that $L$ is an isometry on $L^{2}$, we obtain for all $t$

$$
\int_{\Omega} \frac{1}{2} \psi_{\varepsilon, \delta}^{2}(t)+\frac{1}{2}\left|\nabla q_{\varepsilon, \delta}\right|^{2}(t)=\int_{\Omega} \frac{1}{2} \psi_{\varepsilon, \delta}^{2}(0)+\frac{1}{2}\left|\nabla q_{\varepsilon, \delta}\right|^{2}(0) .
$$

Next, the weak formulation of the conservation of mass yields for almost all $t$

$$
\int_{\Omega} \psi_{\varepsilon, \delta} \varphi_{\varepsilon}(t)+\frac{1}{\varepsilon} \int_{0}^{t} \int_{\Omega} \operatorname{div}\left(\rho_{\varepsilon} u_{\varepsilon}\right) \psi_{\varepsilon, \delta}+\operatorname{div}\left(\nabla q_{\varepsilon, \delta}\right) \varphi_{\varepsilon}=\int_{\Omega} \psi_{\varepsilon, \delta} \varphi_{\varepsilon}(0),
$$

while the weak formulation of (4) implies that we have for almost all $t$

$$
\begin{gathered}
\int_{\Omega} \rho_{\varepsilon} u_{\varepsilon} \cdot v(t)+\int_{0}^{t} \int_{\Omega} \rho_{\varepsilon} u_{\varepsilon} \cdot(v \cdot \nabla v+\nabla p) \\
-\int_{0}^{t} \int_{\Omega} \rho_{\varepsilon} u_{\varepsilon} u_{\varepsilon} \cdot \nabla v+\mu_{\varepsilon} \int_{0}^{t} \int_{\Omega} \nabla u_{\varepsilon} \cdot \nabla v=\int_{\Omega} \rho_{\varepsilon}^{0} u_{\varepsilon}^{0} \cdot u^{0}, \\
\int_{\Omega} \rho_{\varepsilon} u_{\varepsilon} \cdot \nabla q_{\varepsilon, \delta}(t)+\int_{0}^{t} \int_{\Omega} \rho_{\varepsilon} u_{\varepsilon} \cdot\left(\frac{1}{\varepsilon} \nabla \psi_{\varepsilon, \delta}\right)-\int_{0}^{t} \int_{\Omega} \rho_{\varepsilon} u_{\varepsilon} u_{\varepsilon} \cdot \nabla m_{\varepsilon, \delta} \\
+\int_{0}^{t} \int_{\Omega} \mu_{\varepsilon} \nabla u_{\varepsilon} \cdot \nabla m_{\varepsilon, \delta}+\xi_{\varepsilon} \operatorname{div}\left(u_{\varepsilon}\right) \operatorname{div}\left(m_{\varepsilon, \delta}\right) \\
-\int_{0}^{t} \int_{\Omega}\left(\frac{1}{\varepsilon} \varphi_{\varepsilon}+\frac{\gamma-1}{2} \Phi_{\varepsilon}^{2}\right) \operatorname{div}\left(\nabla q_{\varepsilon, \delta}\right)=\int_{\Omega} \rho_{\varepsilon}^{0} u_{\varepsilon}^{0} \cdot m_{\varepsilon, \delta}^{0} .
\end{gathered}
$$

Summing up (14), (15), (16) and subtracting (17), (18), (19), we deduce from straightforward computations the following inequality 
N. MASMOUDI / Ann. Inst. Henri Poincaré, Anal. non linéaire 18 (2001) 199-224

$$
\begin{aligned}
\frac{1}{2} \int_{\Omega} \mid & \sqrt{\rho_{\varepsilon}} u_{\varepsilon}-v-\left.m_{\varepsilon, \delta}\right|^{2}(t)+\left(\Phi_{\varepsilon}-\psi_{\varepsilon, \delta}\right)^{2}(t)+\int_{0} \int_{\Omega} \mu_{\varepsilon}\left|\nabla u_{\varepsilon}\right|^{2}+\xi_{\varepsilon}\left|\operatorname{div}\left(u_{\varepsilon}\right)\right|^{2} \\
\leqslant & -\int_{\Omega}\left(\Phi_{\varepsilon}-\varphi_{\varepsilon}\right) \psi_{\varepsilon, \delta}(t)+\int_{\Omega}\left(\Phi_{\varepsilon}-\varphi_{\varepsilon}\right) \psi_{\varepsilon, \delta}(0) \\
& +\int_{\Omega}\left(\sqrt{\rho_{\varepsilon}}-1\right) \sqrt{\rho_{\varepsilon}} u_{\varepsilon} \cdot\left(v+m_{\varepsilon, \delta}\right)(t) \\
& -\left(\sqrt{\rho_{\varepsilon}^{0}}-1\right) \sqrt{\rho_{\varepsilon}^{0}} u_{\varepsilon}^{0} \cdot\left(v^{0}+m_{\varepsilon, \delta}^{0}\right)-\int_{0}^{t} \int_{\Omega} \mu_{\varepsilon}\left|\nabla u_{\varepsilon}\right|^{2}+\xi_{\varepsilon}\left|\operatorname{div}\left(u_{\varepsilon}\right)\right|^{2} \\
& +\int_{0}^{t} \int_{\Omega} \mu_{\varepsilon} \nabla u_{\varepsilon} \cdot \nabla\left(v+m_{\varepsilon, \delta}\right)+\xi_{\varepsilon} \operatorname{div}\left(u_{\varepsilon}\right) \operatorname{div}\left(m_{\varepsilon, \delta}\right) \\
& +\int_{0}^{t} \int_{\Omega}^{t} \rho_{\varepsilon} u_{\varepsilon} \cdot(v \cdot \nabla v+\nabla p)-\rho_{\varepsilon} u_{\varepsilon}\left(u_{\varepsilon} \cdot \nabla v\right) \\
& -\int_{0}^{t} \int_{\Omega} \rho_{\varepsilon} u_{\varepsilon}\left(u_{\varepsilon} \cdot \nabla m_{\varepsilon, \delta}\right)-\left(\frac{\gamma-1}{2} \Phi_{\varepsilon}^{2}\right) \operatorname{div}\left(\nabla q_{\varepsilon, \delta}\right) \\
& +\frac{1}{2} \int_{\Omega}\left|\sqrt{\rho_{\varepsilon}^{0}} u_{\varepsilon}^{0}-v^{0}-m_{\varepsilon, \delta}^{0}\right|^{2}+\left(\Phi_{\varepsilon}-\psi_{\varepsilon, \delta}\right)^{2}(0) . \\
& \\
& \\
&
\end{aligned}
$$

Only the term marked by $(*)$ in the second hand side of (20) needs some special treatment, we are going to compute it below. In the sequel, we denote by $w_{\varepsilon, \delta}=$ $\sqrt{\rho_{\varepsilon}} u_{\varepsilon}-v-m_{\varepsilon, \delta}$. Then, we have easily

$$
\begin{aligned}
\int_{0}^{t} \int_{\Omega} \rho_{\varepsilon} u_{\varepsilon} \cdot(v \cdot \nabla v+\nabla p)-\rho_{\varepsilon} u_{\varepsilon}\left(u_{\varepsilon} \cdot \nabla v\right) \\
=-\int_{0}^{t} \int_{\Omega} w_{\varepsilon, \delta} \cdot \nabla v w_{\varepsilon, \delta}+\int_{0}^{t} \int_{\Omega}\left(\rho_{\varepsilon}-\sqrt{\rho_{\varepsilon}}\right) u_{\varepsilon} \cdot(v \cdot \nabla v)+\rho_{\varepsilon} u_{\varepsilon} \cdot \nabla p \\
\quad-\left(\sqrt{\rho_{\varepsilon}} u_{\varepsilon}-v\right) \cdot \nabla \frac{v^{2}}{2}-\int_{0}^{t} \int_{\Omega} m_{\varepsilon, \delta} \cdot \nabla v w_{\varepsilon, \delta}+\left(\sqrt{\rho_{\varepsilon}} u_{\varepsilon}-v\right) \cdot \nabla v \cdot m_{\varepsilon, \delta} .
\end{aligned}
$$

Finally, we can see that (20) may be rewritten as

$$
\begin{aligned}
& \left\|w_{\varepsilon, \delta}(t)\right\|_{L^{2}}^{2}+\left\|\Phi_{\varepsilon}-\psi_{\varepsilon, \delta}(t)\right\|_{L^{2}}^{2} \\
& \quad \leqslant\left\|w_{\varepsilon, \delta}(0)\right\|_{L^{2}}^{2}+\left\|\Phi_{\varepsilon}-\psi_{\varepsilon, \delta}(0)\right\|_{L^{2}}^{2}+2 A_{\varepsilon}^{\delta}+2 \int_{0}^{t}\left\|w_{\varepsilon, \delta}(s)\right\|_{L^{2}}^{2}\|\nabla v(s)\|_{L^{\infty}} \mathrm{d} s,
\end{aligned}
$$

where 


$$
\begin{aligned}
A_{\varepsilon}^{\delta}= & -\int_{\Omega}\left(\Phi_{\varepsilon}-\varphi_{\varepsilon}\right) \psi_{\varepsilon, \delta}(t)+\int_{\Omega}\left(\Phi_{\varepsilon}-\varphi_{\varepsilon}\right) \psi_{\varepsilon, \delta}(0) \\
& +\int_{\Omega}\left(\sqrt{\rho_{\varepsilon}}-1\right) \sqrt{\rho_{\varepsilon}} u_{\varepsilon} \cdot\left(v+m_{\varepsilon, \delta}\right)(t)-\left(\sqrt{\rho_{\varepsilon}^{0}}-1\right) \sqrt{\rho_{\varepsilon}^{0}} u_{\varepsilon}^{0} \cdot\left(v^{0}+m_{\varepsilon, \delta}^{0}\right) \\
& +\int_{0}^{t} \int_{\Omega} \mu_{\varepsilon} \nabla u_{\varepsilon} \cdot \nabla\left(v+m_{\varepsilon, \delta}\right)+\xi_{\varepsilon} \operatorname{div}\left(u_{\varepsilon}\right) \operatorname{div}\left(m_{\varepsilon, \delta}\right) \\
& -\int_{0}^{t} \int_{\Omega} \rho_{\varepsilon} u_{\varepsilon}\left(u_{\varepsilon} \cdot \nabla m_{\varepsilon, \delta}\right)-\left(\frac{\gamma-1}{2} \Phi_{\varepsilon}^{2}\right) \operatorname{div}\left(\nabla q_{\varepsilon, \delta}\right) \\
& +\int_{0}^{t} \int_{\Omega}\left(\rho_{\varepsilon}-\sqrt{\rho_{\varepsilon}}\right) u_{\varepsilon} \cdot(v \cdot \nabla v)+\rho_{\varepsilon} u_{\varepsilon} \cdot \nabla p-\left(\sqrt{\rho_{\varepsilon}} u_{\varepsilon}-v\right) \cdot \nabla \frac{v^{2}}{2} \\
& -\int_{0}^{t} \int_{\Omega} m_{\varepsilon, \delta} \cdot \nabla v w_{\varepsilon, \delta}+\left(\sqrt{\rho_{\varepsilon}} u_{\varepsilon}-v\right) \cdot \nabla v \cdot m_{\varepsilon, \delta} .
\end{aligned}
$$

For all fixed $\delta$, it is easy to see that $A_{\varepsilon}^{\delta}(t)$ converges to 0 for almost all $t$, uniformly in $t$ when $\varepsilon$ goes to 0 . Then, by Grönwall's inequality, we deduce that we have for almost all $t \in(0, T)$

$$
\begin{aligned}
& \left\|w_{\varepsilon, \delta}(t)\right\|_{L^{2}}^{2}+\left\|\Phi_{\varepsilon}-\psi_{\varepsilon, \delta}(t)\right\|_{L^{2}}^{2} \\
& \quad \leqslant\left[\left\|w_{\varepsilon, \delta}(0)\right\|_{L^{2}}^{2}+\left\|\Phi_{\varepsilon}-\psi_{\varepsilon, \delta}(0)\right\|_{L^{2}}^{2}+\sup _{0 \leqslant s \leqslant t} A_{\varepsilon}^{\delta}(s)\right] \mathrm{e}^{C \int_{0}^{t}\|\nabla v(s)\|_{L^{\infty}}^{2} .}
\end{aligned}
$$

Then, letting $\varepsilon$ go to 0 , we obtain

$$
\begin{aligned}
\|u-v\|_{L^{\infty}\left(0, T ; L^{2}(\Omega)\right)}^{2} & \leqslant \varlimsup_{\varepsilon}\left(\left\|w_{\varepsilon, \delta}\right\|_{L^{\infty}\left(0, T ; L^{2}(\Omega)\right)}^{2}+\left\|\Phi_{\varepsilon}-\psi_{\varepsilon, \delta}\right\|_{L^{\infty}\left(0, T ; L^{2}(\Omega)\right)}^{2}\right) \\
& \leqslant C_{0}\left(\left\|\tilde{u}^{0}-u^{0}-Q \tilde{u}^{0} * \chi_{\delta}\right\|_{L^{2}(\Omega)}+\left\|\varphi^{0}-\varphi^{0} * \chi_{\delta}\right\|_{L^{2}(\Omega)}\right),
\end{aligned}
$$

where

$$
C_{0}=\mathrm{e}^{C} \int_{0}^{T}\|\nabla v(s)\|_{L^{\infty}}^{2}<+\infty .
$$

Then, letting $\delta$ go to 0 , we deduce that $u=v$ and we obtain also the uniform convergence in $t$ of $P\left(\sqrt{\rho_{\varepsilon}} u_{\varepsilon}\right)$ to $v$ in $L^{2}$, since

$$
\begin{aligned}
& \varlimsup_{\varepsilon}\left\|P\left(\sqrt{\rho_{\varepsilon}} u_{\varepsilon}\right)-v\right\|_{L^{\infty}\left(0, T ; L^{2}\right)} \\
& \quad \leqslant C_{0} \lim _{\delta}\left[\left\|\tilde{u}^{0}-u^{0}-Q \tilde{u}^{0} * \chi_{\delta}\right\|_{L^{2}(\Omega)}+\left\|\varphi^{0}-\varphi^{0} * \chi_{\delta}\right\|_{L^{2}(\Omega)}\right]=0 .
\end{aligned}
$$

Moreover, we see that $\sqrt{\rho_{\varepsilon}} u_{\varepsilon}-m_{\varepsilon}$ converges uniformly in $t$, strongly in $L^{2}$ to $v$. In fact

$$
\left\|\sqrt{\rho_{\varepsilon}} u_{\varepsilon}-m_{\varepsilon}-v\right\|_{L^{2}} \leqslant\left\|\sqrt{\rho_{\varepsilon}} u_{\varepsilon}-m_{\varepsilon, \delta}-v\right\|_{L^{2}}+\left\|m_{\varepsilon}-m_{\varepsilon, \delta}\right\|_{L^{2}}+\left\|\Phi_{\varepsilon}-\psi_{\varepsilon, \delta}\right\|_{L^{2}},
$$


and since $\left(\Phi_{\varepsilon}^{0}, m_{\varepsilon}^{0}=Q \sqrt{\rho_{\varepsilon}^{0}} u_{\varepsilon}^{0}\right)$ converges strongly to $\left(\varphi^{0}, m^{0}\right)$ and since $\mathcal{L}$ is an isometry in $L^{2}$, we deduce that we have

$$
\left\|m_{\varepsilon}-m_{\varepsilon, \delta}\right\|_{L^{2}}+\left\|\Phi_{\varepsilon}-\psi_{\varepsilon, \delta}\right\|_{L^{2}} \rightarrow 0 \quad \text { when } \delta \rightarrow 0
$$

uniformly in $t$ and $\varepsilon$.

Finally, we can also deduce the local strong convergence of $\sqrt{\rho_{\varepsilon}} u_{\varepsilon}$ to $v$ in $L^{p}\left(0, T ; L^{2}(B)\right)$. Indeed let us denote by $B$ a bounded domain of $\mathbb{R}^{N}$. Then, we have for all $t$

$$
\begin{aligned}
\left\|\sqrt{\rho_{\varepsilon}} u_{\varepsilon}-v\right\|_{L^{2}(B)} & \leqslant\left\|\sqrt{\rho_{\varepsilon}} u_{\varepsilon}-m_{\varepsilon, \delta}-v\right\|_{L^{2}(B)}+\left\|m_{\varepsilon, \delta}\right\|_{L^{2}(B)} \\
& \leqslant\left\|\sqrt{\rho_{\varepsilon}} u_{\varepsilon}-m_{\varepsilon, \delta}-v\right\|_{L^{2}(B)}+\left\|m_{\varepsilon, \delta}\right\|_{L^{q}(B)}
\end{aligned}
$$

for any $q>2$. Then, using the fact that for all $\delta,\left\|m_{\varepsilon, \delta}\right\|_{L^{p}\left(0, T ; L^{q}(B)\right)}$ converges to 0 as $\varepsilon$ goes to 0 , we conclude easily by taking the limit in $\varepsilon$ and then in $\delta$ as above.

\section{The periodic case}

As in the case of the whole space, we can deduce, using the energy bounds, that $\rho_{\varepsilon}-1$ converges strongly to 0 in $L^{\infty}\left(0, T ; L^{\gamma}\right)$. Then, using the bound on $\sqrt{\rho_{\varepsilon}} u_{\varepsilon}$ in $L^{\infty}\left(0, T ; L^{2}\right)$, we may extract a subsequence which converges weakly to some $u$. To pass to the limit in the equation, we need to describe the oscillations in time and show that they will not affect the limit equation.

We next introduce, as in the whole space case, the following group $(\mathcal{L}(\tau), \tau \in \mathbb{R})$ defined by $\mathrm{e}^{\tau L}$ where $L$ is the operator defined on $\mathcal{D}_{0}^{\prime} \times\left(\mathcal{D}^{\prime}\right)^{N}$, where $\mathcal{D}_{0}^{\prime}=\{\varphi \in$ $\left.\mathcal{D}^{\prime}, \int \varphi=0\right\}$, by

$$
L\left(\begin{array}{l}
\varphi \\
v
\end{array}\right)=-\left(\begin{array}{c}
\operatorname{div} v \\
\nabla \varphi
\end{array}\right) .
$$

In the sequel, we will use the following notations

$$
U^{\varepsilon}=\left(\varphi_{\varepsilon}, Q\left(\rho_{\varepsilon} u_{\varepsilon}\right)\right) \quad \text { and } \quad V^{\varepsilon}=\mathcal{L}(-t / \varepsilon)\left(\varphi_{\varepsilon}, Q\left(\rho_{\varepsilon} u_{\varepsilon}\right)\right),
$$

and for some technical reasons related to the $L^{2}$ integrability, we will also use the following approximations

$$
\bar{U}^{\varepsilon}=\left(\Phi_{\varepsilon}, Q\left(\sqrt{\rho_{\varepsilon}} u_{\varepsilon}\right)\right) \quad \text { and } \quad \bar{V}^{\varepsilon}=\mathcal{L}(-t / \varepsilon)\left(\Phi_{\varepsilon}, Q\left(\sqrt{\rho_{\varepsilon}} u_{\varepsilon}\right)\right),
$$

which satisfy

$$
\left\|U^{\varepsilon}-\bar{U}^{\varepsilon}\right\|_{L^{\infty}\left(L^{2 \gamma /(\gamma+1)}\right)} \rightarrow 0 \quad \text { when } \varepsilon \rightarrow 0 .
$$

Let us project Eq. (4) on "gradient vector-fields"

$$
\begin{gathered}
\frac{\partial}{\partial t} Q\left(\rho_{\varepsilon} u_{\varepsilon}\right)+Q\left[\operatorname{div}\left(\rho_{\varepsilon} u_{\varepsilon} \otimes u_{\varepsilon}\right)\right]-\left(\mu_{\varepsilon}+\xi_{\varepsilon}\right) \nabla \operatorname{div} u_{\varepsilon} \\
+\frac{a}{\varepsilon^{2}} \nabla\left(\rho_{\varepsilon}^{\gamma}-\gamma \rho_{\varepsilon}+(\gamma-1)\right)+\frac{1}{\varepsilon^{2}} \nabla\left(\rho_{\varepsilon}-1\right)=0 .
\end{gathered}
$$


Combining (26) with the conservation of mass, we obtain

$$
\varepsilon \frac{\partial \varphi_{\varepsilon}}{\partial t}+\operatorname{div} Q\left(\rho_{\varepsilon} u_{\varepsilon}\right)=0, \quad \varepsilon \frac{\partial}{\partial t} Q\left(\rho_{\varepsilon} u_{\varepsilon}\right)+\nabla \varphi_{\varepsilon}=\varepsilon F_{\varepsilon},
$$

where

$$
F_{\varepsilon}=\left(\mu_{\varepsilon}+\xi_{\varepsilon}\right) \nabla \operatorname{div} u_{\varepsilon}-Q\left[\operatorname{div}\left(\rho_{\varepsilon} u_{\varepsilon} \otimes u_{\varepsilon}\right)\right]-a \nabla\left[\frac{1}{\varepsilon^{2}}\left(\rho_{\varepsilon}^{\gamma}-\gamma \rho_{\varepsilon}+(\gamma-1)\right)\right] .
$$

Eq. (27) yields that $\partial_{t} U^{\varepsilon}=\frac{1}{\varepsilon} L U^{\varepsilon}+\left(0, F_{\varepsilon}\right)$, which can be rewritten as $\partial_{t} V^{\varepsilon}=$ $\mathcal{L}(-t / \varepsilon)\left(0, F_{\varepsilon}\right)$. It is easy to check that $F_{\varepsilon}$ is bounded in $L^{2}\left(H^{-s}\right)$ (for some $s \in \mathbb{R}$ ), hence $V^{\varepsilon}$ is compact in time (the oscillations have been cancelled). If we had enough compactness in space we could pass to the limit in this equation and recover the following limit system for the oscillating part

$$
\partial_{t} \bar{V}+\mathcal{Q}_{1}(u, \bar{V})+\mathcal{Q}_{2}(\bar{V}, \bar{V})-v \Delta \bar{V}=0,
$$

where $\mathcal{Q}_{1}$ and $\mathcal{Q}_{2}$ are respectively a linear and a bilinear forms in $\bar{V}$ (which will be defined and computed later on) and the term $-v \Delta \bar{V}$ explained below. In fact, as in [17] (see also [16]), we consider $\mathcal{L}(-t / \varepsilon)\left(0, F_{\varepsilon}\right)$ as an almost periodic function in $\tau=t / \varepsilon$ and compute its mean value, which yields (28).

DEFINITION 3.1. - For all divergence-free vector field $u \in L^{2}(\Omega)^{N}$ and all $V=$ $(\psi, \nabla q) \in L^{2}(\Omega)^{N+1}$, we define the following linear and bilinear symmetric forms in V

$$
\mathcal{Q}_{1}(u, V)=\lim _{\tau \rightarrow \infty} \frac{1}{\tau} \int_{0}^{\tau} \mathcal{L}(-s)\left(\begin{array}{c}
0 \\
\operatorname{div}\left(u \otimes \mathcal{L}_{2}(s) V+\mathcal{L}_{2}(s) V \otimes u\right)
\end{array}\right) \mathrm{d} s,
$$

and

$$
\mathcal{Q}_{2}(V, V)=\lim _{\tau \rightarrow \infty} \frac{1}{\tau} \int_{0}^{\tau} \mathcal{L}(-s)\left(\begin{array}{c}
0 \\
\operatorname{div}\left(\mathcal{L}_{2}(s) V \otimes \mathcal{L}_{2}(s) V\right)+\frac{\gamma-1}{2} \nabla\left(\mathcal{L}_{1}(s) V\right)^{2}
\end{array}\right) \mathrm{d} s .
$$

The convergences stated above takes place in $W^{-1,1}$ and can be shown by using almost-periodic functions (see [16] and the references therein). Indeed the functions inside the integral in (29) and (30) are almost periodic in $W^{-1,1}$ and $\mathcal{Q}_{1}(u, V), \mathcal{Q}_{2}(V, V)$ are their mean value. We will come back to this issue in the next section. We also remark that in Eq. (28) the viscosity term was divided by 2 and that it applies for both component of the vector $\bar{V}$. This is due to the following proposition, which can be proved easily using almost periodic functions (see also [5] and [3]).

PROPOSITION 3.2. - Under the same hypothesis on $V$, we have

$$
-v \Delta V=\lim _{\tau \rightarrow \infty} \frac{1}{\tau} \int_{0}^{\tau}-\mathcal{L}(-s)\left(\begin{array}{c}
0 \\
2 v \Delta \mathcal{L}_{2}(s) V
\end{array}\right) \mathrm{d} s .
$$


Nevertheless, the fact that the viscosity applies now for both components of $V$ is not sufficient to yield compactness in space for $V^{\varepsilon}$. To recover compactness in space, we will use the regularity of the limit system and extend the method used in [12] to the case of general initial data as was done in [16]. Let $V^{0}$ be the solution of the following system

$$
\left\{\begin{array}{l}
\partial_{t} V^{0}+\mathcal{Q}_{1}\left(v, V^{0}\right)+\mathcal{Q}_{2}\left(V^{0}, V^{0}\right)-v \Delta V^{0}=0 \\
V_{\mid t=0}^{0}=\left(\varphi^{0}, Q \tilde{u}^{0}\right)
\end{array}\right.
$$

where $v$ is, as in the case of the whole space, the solution of the incompressible Euler equations with initial data $u^{0}$. The existence of global strong solutions for the system (32) (and local solutions if the viscosity term is removed) as well as the exact computations of the two forms $\mathcal{Q}_{1}$ and $\mathcal{Q}_{2}$ will be detailed in the next section. We only need the following two propositions.

PROpOSITION 3.3. - For all $u, V, V_{1}$ and $V_{2}$ (regular enough to define all the products), we have

$$
\begin{gathered}
\int \mathcal{Q}_{1}(u, V) V=0 \quad \text { and } \int \mathcal{Q}_{2}(V, V) V=0, \\
\int \mathcal{Q}_{1}\left(u, V_{1}\right) V_{2}+\mathcal{Q}_{1}\left(u, V_{2}\right) V_{1}=0, \\
\int \mathcal{Q}_{2}\left(V_{1}, V_{1}\right) V_{2}+2 \mathcal{Q}_{2}\left(V_{1}, V_{2}\right) V_{1}=0 .
\end{gathered}
$$

The proof of (33) will be given in the next section, (34) can be shown by applying the first part of (33) to $V_{1}+V_{2}$ and to $V_{1}-V_{2}$. Finally (35) can be shown by applying the second part of (33) to $V_{1}+X V_{2}$ and identifying the term of degree 1.

The next proposition is a very simple consequence of the theory of almost-periodic functions (see for instance Lemma 2.3 of [16]).

Proposition 3.4. - For all $u \in L^{p}\left(0, T ; L^{2}\right)$ and $V \in L^{q}\left(0, T ; L^{2}\right)$, we have the following weak convergences ( $p$ and $q$ are such that the product are well defined)

$$
w-\lim _{\varepsilon} \mathcal{L}\left(-\frac{t}{\varepsilon}\right)\left(\begin{array}{c}
0 \\
\operatorname{div}\left(u \otimes \mathcal{L}_{2}\left(\frac{t}{\varepsilon}\right) V+\mathcal{L}_{2}\left(\frac{t}{\varepsilon}\right) V \otimes u\right)
\end{array}\right)=\mathcal{Q}_{1}(u, V)
$$

and

$$
w-\lim _{\varepsilon} \mathcal{L}\left(-\frac{t}{\varepsilon}\right)\left(\begin{array}{c}
0 \\
\operatorname{div}\left(\mathcal{L}_{2}\left(\frac{t}{\varepsilon}\right) V \otimes \mathcal{L}_{2}\left(\frac{t}{\varepsilon}\right) V\right)+\frac{\gamma-1}{2} \nabla\left(\mathcal{L}_{1}\left(\frac{t}{\varepsilon}\right) V\right)^{2}
\end{array}\right)=\mathcal{Q}_{2}(V, V) .
$$

Using the symmetry of $Q_{2}$, we deduce easily the following proposition.

Proposition 3.5. - Eq. (37) of Proposition 3.4 can be extended to the case where we take $V_{1}$ and $V_{2}$ using the symmetry of $Q_{2}$, namely 


$$
\begin{aligned}
& w-\lim _{\varepsilon} \mathcal{L}\left(-\frac{t}{\varepsilon}\right)\left(\begin{array}{c}
0 \\
\operatorname{div}\left(\mathcal{L}_{2}\left(\frac{t}{\varepsilon}\right) V_{1} \otimes \mathcal{L}_{2}\left(\frac{t}{\varepsilon}\right) V_{2}+\mathcal{L}_{2}\left(\frac{t}{\varepsilon}\right) V_{2} \otimes \mathcal{L}_{2}\left(\frac{t}{\varepsilon}\right) V_{1}\right)
\end{array}\right) \\
& +\mathcal{L}\left(-\frac{t}{\varepsilon}\right)\left(\begin{array}{c}
0 \\
\frac{\gamma-1}{2} \nabla\left(\mathcal{L}_{1}\left(\frac{t}{\varepsilon}\right) V_{1} \mathcal{L}_{1}\left(\frac{t}{\varepsilon}\right) V_{2}\right)
\end{array}\right)=\mathcal{Q}_{2}\left(V_{1}, V_{2}\right) .
\end{aligned}
$$

Moreover, the above identity holds for $V_{1} \in L^{q}\left(0, T ; H^{s}\right)$ and $V_{2} \in L^{p}\left(0, T ; H^{-s}\right)$ with $s \in \mathbb{R}$ and $1 / p+1 / q=1$. It is also possible to extend it to the case where we replace $V_{2}$ in the left hand side by a sequence $V_{2}^{\varepsilon}$ such that $V_{2}^{\varepsilon}$ converges strongly to $V_{2}$ in $L^{p}\left(0, T ; H^{-s}\right)$.

In order to show the convergence in Theorem 1.2, we will try to estimate

$$
\left\|\sqrt{\rho_{\varepsilon}} u_{\varepsilon}-v-\mathcal{L}_{2}\left(\frac{t}{\varepsilon}\right) V^{0}\right\|_{L^{2}}^{2}+\left\|\Phi_{\varepsilon}-\mathcal{L}_{1}\left(\frac{t}{\varepsilon}\right) V^{0}\right\|_{L^{2}}^{2} .
$$

We also introduce the following notations $w_{\varepsilon}=\sqrt{\rho_{\varepsilon}} u_{\varepsilon}-v-\mathcal{L}_{2}(t / \varepsilon) V^{0}$ and $\beta_{\varepsilon}=$ $\Phi_{\varepsilon}-\mathcal{L}_{1}(t / \varepsilon) V^{0}$. In the sequel, we also note $\left(\psi_{\varepsilon}, m_{\varepsilon}\right)=\mathcal{L}(t / \varepsilon) V^{0}$. The proof follows the same lines as the proof in the whole space case apart from the fact that the equation satisfied by the gradient part is not trivial and that we have to use the precise equation satisfied by the oscillating terms. We recall the energy inequality

$$
\frac{1}{2} \int_{\Omega} \rho_{\varepsilon}\left|u_{\varepsilon}\right|^{2}(t)+\Phi_{\varepsilon}^{2}(t)+\int_{0}^{t} \int_{\Omega} \mu_{\varepsilon}\left|D u_{\varepsilon}\right|^{2}+\xi_{\varepsilon}\left(\operatorname{div} u_{\varepsilon}\right)^{2} \leqslant \frac{1}{2} \int_{\Omega} \rho_{\varepsilon}^{0}\left|u_{\varepsilon}^{0}\right|^{2}+\Phi_{\varepsilon}^{2}(0)
$$

as well as the conservation of energy for $v$

$$
\int_{\Omega} \frac{1}{2}|v|^{2}(t)=\int_{\Omega} \frac{1}{2}\left|u_{0}\right|^{2}
$$

Using that

$$
\int \mathcal{Q}_{1}\left(u, V^{0}\right) V^{0}=0, \quad \int \mathcal{Q}_{2}\left(V^{0}, V^{0}\right) V^{0}=0,
$$

we deduce from (32) the following energy identity

$$
\int_{\Omega} \frac{1}{2}\left|V^{0}\right|^{2}(t)+v \int_{0}^{t} \int_{\Omega}\left|\nabla V^{0}\right|^{2}(s) \mathrm{d} s=\int_{\Omega} \frac{1}{2}\left|V^{0}(t=0)\right|^{2} .
$$

Next, using the weak formulation of the conservation of mass, we obtain for almost all $t$

$$
\int_{\Omega} \psi_{\varepsilon} \varphi_{\varepsilon}(t)+\frac{1}{\varepsilon} \int_{0}^{t} \int_{\Omega} \operatorname{div}\left(\rho_{\varepsilon} u_{\varepsilon}\right) \psi_{\varepsilon}+\operatorname{div}\left(\nabla q_{\varepsilon}\right) \varphi_{\varepsilon}
$$


N. MASMOUDI / Ann. Inst. Henri Poincaré, Anal. non linéaire 18 (2001) 199-224

$$
-\int_{0}^{t} \int_{\Omega} \mathcal{L}_{1}\left(\frac{t}{\varepsilon}\right) \partial_{t} V^{0} \varphi_{\varepsilon}=\int_{\Omega} \varphi^{0} \varphi_{\varepsilon}(0),
$$

while the weak formulation of (4), yields as before the following inequality for almost all $t$

$$
\begin{gathered}
\int_{\Omega} \rho_{\varepsilon} u_{\varepsilon} \cdot v(t)+\int_{0} \int_{\Omega} \rho_{\varepsilon} u_{\varepsilon} \cdot(v \cdot \nabla v+\nabla p) \\
-\int_{0}^{t} \int_{\Omega} \rho_{\varepsilon} u_{\varepsilon} u_{\varepsilon} \cdot \nabla v+\mu_{\varepsilon} \int_{0}^{t} \int_{\Omega} \nabla u_{\varepsilon} \cdot \nabla v=\int_{\Omega} \rho_{\varepsilon}^{0} u_{\varepsilon}^{0} \cdot u^{0}, \\
\int_{\Omega} \rho_{\varepsilon} u_{\varepsilon} \cdot \nabla q_{\varepsilon}(t)+\int_{0}^{t} \int_{\Omega} \rho_{\varepsilon} u_{\varepsilon} \cdot\left(\frac{1}{\varepsilon} \nabla \psi_{\varepsilon}\right)-\int_{0}^{t} \int_{\Omega} \rho_{\varepsilon} u_{\varepsilon} u_{\varepsilon} \cdot \nabla m_{\varepsilon} \\
-\int_{0}^{t} \int_{\Omega} \mathcal{L}_{2}\left(\frac{t}{\varepsilon}\right) \partial_{t} V^{0} \rho_{\varepsilon} u_{\varepsilon}+\int_{0}^{t} \int_{\Omega} \mu_{\varepsilon} \nabla u_{\varepsilon} \cdot \nabla m_{\varepsilon}+\xi_{\varepsilon} \operatorname{div}\left(u_{\varepsilon}\right) \operatorname{div}\left(m_{\varepsilon}\right) \\
-\int_{0}^{t} \int_{\Omega}\left(\frac{1}{\varepsilon} \varphi_{\varepsilon}+\frac{\gamma-1}{2} \Phi_{\varepsilon}^{2}\right) \operatorname{div}\left(\nabla q_{\varepsilon}\right)=\int_{\Omega} \rho_{\varepsilon}^{0} u_{\varepsilon}^{0} \cdot Q \tilde{u}^{0} .
\end{gathered}
$$

Next adding up (39), (40), (41) and subtracting (42), (43), (44), we obtain

$$
\begin{aligned}
& \frac{1}{2} \int_{\Omega}\left|w_{\varepsilon}\right|^{2}(t)+\left(\beta_{\varepsilon}\right)^{2}(t)+\int_{0}^{t} \int_{\Omega} \mu_{\varepsilon}\left|D w_{\varepsilon}\right|^{2}+\xi_{\varepsilon}\left(\operatorname{div} w_{\varepsilon}\right)^{2} \\
& \leqslant-\int_{\Omega}\left(\Phi_{\varepsilon}-\varphi_{\varepsilon}\right) \psi_{\varepsilon}(t)+\int_{\Omega}\left(\Phi_{\varepsilon}-\varphi_{\varepsilon}\right) \varphi^{0} \\
& \quad+\int_{\Omega}\left(\sqrt{\rho_{\varepsilon}}-1\right) \sqrt{\rho_{\varepsilon}} u_{\varepsilon} \cdot\left(v+m_{\varepsilon}\right)(t)-\left(\sqrt{\rho_{\varepsilon}^{0}}-1\right) \sqrt{\rho_{\varepsilon}^{0}} u_{\varepsilon}^{0} \cdot \tilde{u}^{0} \\
& \quad+\frac{1}{2} \int_{\Omega}\left|\sqrt{\rho_{\varepsilon}^{0}} u_{\varepsilon}^{0}-\tilde{u}^{0}\right|^{2}+\left(\Phi_{\varepsilon}-\varphi^{0}\right)^{2}(0)+A_{\varepsilon}+B_{\varepsilon},
\end{aligned}
$$

where $A_{\varepsilon}$ and $B_{\varepsilon}$ are given by

$$
\begin{aligned}
A_{\varepsilon}= & \int_{0}^{t} \int_{\Omega}-v\left|\nabla V^{0}\right|^{2}+\mu_{\varepsilon}\left|D m_{\varepsilon}\right|^{2}+\xi_{\varepsilon}\left(\operatorname{div} m_{\varepsilon}\right)^{2}+\mu_{\varepsilon}|D v|^{2} \\
& +\int_{0}^{t} \int_{\Omega}-\mu_{\varepsilon} \nabla u_{\varepsilon} \cdot \nabla\left(v+m_{\varepsilon}\right)-\xi_{\varepsilon}\left(\operatorname{div} u_{\varepsilon}\right)\left(\operatorname{div} m_{\varepsilon}\right)-v \Delta V^{0} V^{\varepsilon}
\end{aligned}
$$

and 


$$
\begin{aligned}
B_{\varepsilon}= & \int_{0}^{t} \int_{\Omega} \rho_{\varepsilon} u_{\varepsilon} \cdot(v \cdot \nabla v+\nabla p)-\rho_{\varepsilon} u_{\varepsilon} u_{\varepsilon} \cdot \nabla\left(v+m_{\varepsilon}\right) \\
& +\int_{0}^{t} \int_{\Omega}-\frac{\gamma-1}{2} \Phi_{\varepsilon}^{2} \operatorname{div}\left(m_{\varepsilon}\right)+\mathcal{Q}_{1}\left(v, V^{0}\right) V^{\varepsilon}+\mathcal{Q}_{2}\left(V^{0}, V^{0}\right) V^{\varepsilon} .
\end{aligned}
$$

Here, we have used that

$$
\int_{0}^{t} \int_{\Omega} \mathcal{L}\left(\frac{t}{\varepsilon}\right) \partial_{t} V^{0} U^{\varepsilon}=\int_{0}^{t} \int_{\Omega} \partial_{t} V^{0} V^{\varepsilon} .
$$

It is easy to see that to apply a Grönwall's lemma, one has to estimate $A_{\varepsilon}$ and $B_{\varepsilon}$, since one can show (as in the case of the whole space) that the other terms in the right hand side of (45) converge to 0 uniformly in t. In the following two subsections, we will show that

$$
B_{\varepsilon}(t) \leqslant\left(\left\|w_{\varepsilon}(t)\right\|_{L^{2}}^{2}+\left\|\beta_{\varepsilon}(t)\right\|_{L^{2}}^{2}\right)\left\|\nabla\left(v+m_{\varepsilon}\right)\right\|_{L^{\infty}}+r_{\varepsilon}
$$

and that $A_{\varepsilon}, r_{\varepsilon}$ converge to 0 uniformly in t. Therefore, we conclude as in the case of the whole space, using that $\nabla\left(v+m_{\varepsilon}\right)$ is bounded in $L^{1}\left(0, T ; L^{\infty}\right)$ uniformly in $\varepsilon$.

\subsection{Bounds on $B_{\varepsilon}$}

We recall here that we assume (extracting subsequences if necessary) that $\sqrt{\rho_{\varepsilon}} u_{\varepsilon}$ and $u_{\varepsilon}$ converges weakly to some $u$ and that $V^{\varepsilon}=\mathcal{L}(-t / \varepsilon) U^{\varepsilon}$ as well as $\bar{V}^{\varepsilon}=\mathcal{L}(-t / \varepsilon) \bar{U}^{\varepsilon}$ converge (strongly in time) to $(0, u)+\bar{V}$. In the sequel, $r_{\varepsilon}$ will denote any sequence of functions converging uniformly in $\mathrm{t}$ to 0 . Rewriting (47), we get

$$
\begin{aligned}
B_{\varepsilon}= & \int_{0}^{t} \int_{\Omega}-w_{\varepsilon} w_{\varepsilon} \cdot \nabla\left(v+m_{\varepsilon}\right)-\frac{\gamma-1}{2} \beta_{\varepsilon}^{2} \operatorname{div}\left(m_{\varepsilon}\right) \\
& +\int_{0}^{t} \int_{\Omega} \sqrt{\rho_{\varepsilon}} u_{\varepsilon} \cdot(v \cdot \nabla v)-\sqrt{\rho_{\varepsilon}} u_{\varepsilon} \cdot\left(v+m_{\varepsilon}\right) \cdot \nabla\left(v+m_{\varepsilon}\right) \\
& +\int_{0}^{t} \int_{\Omega}-v \cdot\left(\sqrt{\rho_{\varepsilon}} u_{\varepsilon}-v-m_{\varepsilon}\right) \cdot \nabla\left(v+m_{\varepsilon}\right) \\
& -m_{\varepsilon} \cdot\left(\sqrt{\rho_{\varepsilon}} u_{\varepsilon}-v-m_{\varepsilon}\right) \cdot \nabla\left(v+m_{\varepsilon}\right) \\
& +\int_{0}^{t} \int_{\Omega} \frac{\gamma-1}{2} \psi_{\varepsilon}^{2} \operatorname{div}\left(m_{\varepsilon}\right)-2 \frac{\gamma-1}{2} \psi_{\varepsilon} \Phi_{\varepsilon} \operatorname{div}\left(m_{\varepsilon}\right) \\
& +\int_{0}^{t} \int_{\Omega} \mathcal{Q}_{1}\left(v, V^{0}\right) V^{\varepsilon}+\mathcal{Q}_{2}\left(V^{0}, V^{0}\right) V^{\varepsilon}+r_{\varepsilon} .
\end{aligned}
$$


Let us compute the limit when $\varepsilon$ goes to 0 of the terms appearing in the right hand side. On the one hand, we have

$$
\begin{aligned}
& \int_{0}^{t} \int_{\Omega} m_{\varepsilon} \cdot m_{\varepsilon} \cdot \nabla\left(v+m_{\varepsilon}\right)+\frac{\gamma-1}{2} \psi_{\varepsilon}^{2} \operatorname{div}\left(m_{\varepsilon}\right) \\
& =-\int_{0}^{t} \int_{\Omega}\left[\operatorname{div}\left(m_{\varepsilon} \otimes m_{\varepsilon}\right)+\frac{\gamma-1}{2} \nabla\left(\psi_{\varepsilon}^{2}\right)\right] \cdot\left(m_{\varepsilon}+v\right) \\
& =-\int_{0}^{t} \int_{\Omega} \mathcal{L}\left(-\frac{t}{\varepsilon}\right)\left(\begin{array}{c}
0 \\
\operatorname{div}\left(m_{\varepsilon} \otimes m_{\varepsilon}\right)+\frac{\gamma-1}{2} \nabla\left(\psi_{\varepsilon}\right)^{2}
\end{array}\right) \cdot\left(V^{0}+\left(\begin{array}{l}
0 \\
v
\end{array}\right)\right) \\
& =-\int_{0}^{t} \int_{\Omega} \mathcal{Q}_{2}\left(V^{0}, V^{0}\right) \cdot\left(V^{0}+(0, v)\right)+r_{\varepsilon}=r_{\varepsilon} .
\end{aligned}
$$

On the other hand, using Proposition 3.5, we have

$$
\begin{aligned}
& -\int_{0}^{t} \int_{\Omega}\left(m_{\varepsilon} \otimes \sqrt{\rho_{\varepsilon}} u_{\varepsilon}+\sqrt{\rho_{\varepsilon}} u_{\varepsilon} \otimes m_{\varepsilon}\right): \nabla\left(v+m_{\varepsilon}\right)+(\gamma-1) \psi_{\varepsilon} \Phi_{\varepsilon} \operatorname{div}\left(m_{\varepsilon}\right) \\
& =\int_{0}^{t} \int_{\Omega}\left[\operatorname{div}\left(m_{\varepsilon} \otimes \sqrt{\rho_{\varepsilon}} u_{\varepsilon}+\sqrt{\rho_{\varepsilon}} u_{\varepsilon} \otimes m_{\varepsilon}\right)+(\gamma-1) \nabla\left(\psi_{\varepsilon} \Phi_{\varepsilon}\right)\right] .\left(m_{\varepsilon}+v\right)
\end{aligned}
$$

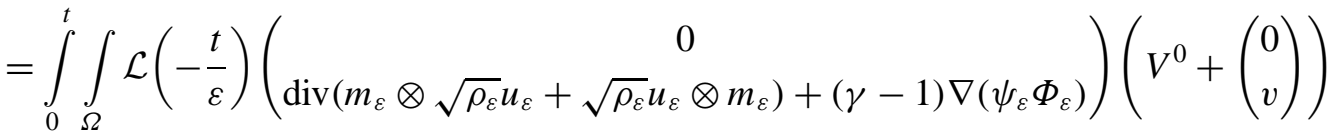

$$
\begin{aligned}
& =\int_{0}^{t} \int_{\Omega} 2 \mathcal{Q}_{2}\left(V^{0}, \bar{V}\right) \cdot\left(V^{0}+(0, v)\right)+\mathcal{Q}_{1}\left(u, V^{0}\right) \cdot\left(V^{0}+(0, v)\right)+r_{\varepsilon} \\
& =2 \int_{0}^{t} \int_{\Omega} \mathcal{Q}_{2}\left(V^{0}, \bar{V}\right) \cdot V^{0}+r_{\varepsilon} .
\end{aligned}
$$

Moreover, it is easy to see that

$$
\int_{0}^{t} \int_{\Omega}\left[\operatorname{div}\left(v \otimes \sqrt{\rho_{\varepsilon}} u_{\varepsilon}+\sqrt{\rho_{\varepsilon}} u_{\varepsilon} \otimes v\right)\right] . m_{\varepsilon}=\int_{0}^{t} \int_{\Omega} \mathcal{Q}_{1}(v, \bar{V}) . V^{0}+r_{\varepsilon},
$$

and that

$$
\int_{0}^{t} \int_{\Omega}\left[\operatorname{div}\left(v \otimes m_{\varepsilon}+m_{\varepsilon} \otimes v\right)\right] . m_{\varepsilon}=\int_{0}^{t} \int_{\Omega} \mathcal{Q}_{1}\left(v, V^{0}\right) \cdot V^{0}+r_{\varepsilon}=r_{\varepsilon} .
$$


Finally, we also have

$$
\int_{0}^{t} \int_{\Omega} \mathcal{Q}_{1}\left(v, V^{0}\right) V^{\varepsilon}+\mathcal{Q}_{2}\left(V^{0}, V^{0}\right) V^{\varepsilon}=\int_{0}^{t} \int_{\Omega} \mathcal{Q}_{1}\left(v, V^{0}\right) \bar{V}+\mathcal{Q}_{2}\left(V^{0}, V^{0}\right) \bar{V}+r_{\varepsilon} .
$$

\subsection{Bounds on $A_{\varepsilon}$}

We recall that $\mu_{\varepsilon}$ goes to 0 and that $\xi_{\varepsilon}$ goes to $2 v$. From the energy bounds on $V^{0}$ and on $v$, we get

$$
\int_{0}^{t} \int_{\Omega} \mu_{\varepsilon}\left|D m_{\varepsilon}\right|^{2}+\mu_{\varepsilon}|D v|^{2}-\mu_{\varepsilon} \nabla u_{\varepsilon} \cdot \nabla\left(v+m_{\varepsilon}\right) \rightarrow 0
$$

uniformly in $t$. Besides, integrating by parts and using that $m_{\varepsilon}=\nabla q_{\varepsilon}$, we get

$$
\begin{aligned}
\int_{0}^{t} \int_{\Omega} \xi_{\varepsilon}\left(\operatorname{div} m_{\varepsilon}\right)^{2} & =-\int_{0}^{t} \int_{\Omega} \xi_{\varepsilon} \Delta m_{\varepsilon} \cdot m_{\varepsilon}=-\int_{0}^{t} \int_{\Omega} \mathcal{L}\left(-\frac{t}{\varepsilon}\right)\left(\begin{array}{c}
0 \\
\xi_{\varepsilon} \Delta m_{\varepsilon}
\end{array}\right) \cdot V^{0} \\
& =-\int_{0}^{t} \int_{\Omega} \nu \Delta V^{0} V^{0}+r_{\varepsilon} .
\end{aligned}
$$

The same argument yields

$$
-\int_{0}^{t} \int_{\Omega} \xi_{\varepsilon}\left(\operatorname{div} m_{\varepsilon}\right)\left(\operatorname{div} u_{\varepsilon}\right)=\int_{0}^{t} \int_{\Omega} \mathcal{L}\left(-\frac{t}{\varepsilon}\right)\left(\begin{array}{c}
0 \\
\xi_{\varepsilon} \Delta u_{\varepsilon}
\end{array}\right) \cdot V^{0}=\int_{0}^{t} \int_{\Omega} v \Delta \bar{V} V^{0}+r_{\varepsilon} .
$$

Finally, we have

$$
\int_{0}^{t} \int_{\Omega}-v \Delta V^{0} V^{\varepsilon}=\int_{0}^{t} \int_{\Omega}-v \Delta V^{0} \bar{V}+r_{\varepsilon}
$$

Adding up the different contributions and integrating by parts, we deduce that $A_{\varepsilon}=r_{\varepsilon}$ converges to 0 uniformly in $t$.

\section{Study of the oscillating part}

This section is devoted to the study of the equation satisfied by the gradient part of the momentum. We expect that the following computations will be used in a forthcoming investigation of a new numerical approach to slightly compressible flows.

\subsection{Computation of $\mathcal{Q}_{2}$}

We recall that $\Omega=\mathbb{T}^{N}$ and that $\mathbb{T}^{N}$ is a periodic domain with periods $\left(2 \pi a_{1}, \ldots\right.$, $\left.2 \pi a_{N}\right)$, where for all $i, a_{i}>0$. Then, we can decompose $L^{2}(\Omega) \times\left\{\nabla q, q \in H^{1}(\Omega)\right\}$ into 
the following orthogonal basis. (In the sequel $L^{2}(\Omega)$ denotes the space of $L^{2}$ functions with zero average)

$$
V_{\mathbf{k}}^{+}(X)=\frac{1}{\sqrt{2\left|\mathbb{T}^{N}\right||\mathbf{k}|}}\left(\begin{array}{c}
|\mathbf{k}| \\
-s g(\mathbf{k}) \mathbf{k}
\end{array}\right) \mathrm{e}^{i \mathbf{k} \cdot X}
$$

and

$$
V_{\mathbf{k}}^{-}(X)=\frac{1}{\sqrt{2\left|\mathbb{T}^{N}\right||\mathbf{k}|}}\left(\begin{array}{c}
|\mathbf{k}| \\
\operatorname{sg}(\mathbf{k}) \mathbf{k}
\end{array}\right) \mathrm{e}^{i \mathbf{k} \cdot X},
$$

where $\mathbf{k}$ is the vector whose components are defined by $\mathbf{k}_{i}=\mathbf{k}_{i}^{\prime} / a_{i}$ for all $1 \leqslant i \leqslant N$ with $\mathbf{k}_{i}^{\prime} \in \mathbb{Z}^{*}$ and where the notation $|\mathbf{k}|$ is defined by $|\mathbf{k}|^{2}=\sum_{i} \mathbf{k}_{i}^{2}$ and $\left|\mathbb{T}^{N}\right|=(2 \pi)^{N} \prod_{i} a_{i}$. Moreover, $s g(\mathbf{k})$ is a generalization of the function sign, defined on $\mathbb{R}^{N}-\{0\}$ by $\operatorname{sg}(\mathbf{k})=+1$ if and only if there exists $i, 1 \leqslant i \leqslant N$, such that for all $j<i$, we have $\mathbf{k}_{j}=0$ and $\mathbf{k}_{i}>0$. Otherwise $s g(\mathbf{k})=-1$. We think that the introduction of this notation yields much simpler formulae. We only point out that we have

$$
L V_{\mathbf{k}}^{+}(X)=i \operatorname{sg}(\mathbf{k})|\mathbf{k}| V_{\mathbf{k}}^{+}(X) \quad \text { and } \quad L V_{\mathbf{k}}^{-}(X)=-i \operatorname{sig}(\mathbf{k})|\mathbf{k}| V_{\mathbf{k}}^{-}(X) \text {. }
$$

Decomposing $V$ on this basis, we have

$$
V=\sum_{\mathbf{k}^{\prime} \in \mathbb{Z}^{N *}} a_{\mathbf{k}}^{+} V_{\mathbf{k}}^{+}+a_{\mathbf{k}}^{-} V_{\mathbf{k}}^{-}
$$

We want to remark here that $V_{\mathbf{k}}^{+}=\overline{V_{-\mathbf{k}}^{+}}$and that $a_{-\mathbf{k}}^{+}=\overline{a_{\mathbf{k}}^{+}}$, since $V$ is a real function. The same holds with + replaced by - . Then, applying the group $\mathcal{L}$, we obtain

$$
\mathcal{L}(s) V=\sum_{\mathbf{k}^{\prime} \in \mathbb{Z}^{N *}} a_{\mathbf{k}}^{+} V_{\mathbf{k}}^{+} \mathrm{e}^{i s g(\mathbf{k})|\mathbf{k}| s}+a_{\mathbf{k}}^{-} V_{\mathbf{k}}^{-} \mathrm{e}^{i s g(\mathbf{k})|\mathbf{k}| s} .
$$

Next, we can compute easily the term inside the integral in the right hand side of (30)

$$
\begin{aligned}
& \mathcal{L}(-s) \sum_{\substack{\mathbf{k}, \mathbf{l} \\
\alpha= \pm, \beta= \pm}} a_{\mathbf{k}}^{\alpha} a_{\mathbf{l}}^{\beta} i\left(\begin{array}{c}
0 \\
(\mathbf{k}+\mathbf{l}) \cdot[\alpha \beta s g(\mathbf{k}) s g(\mathbf{l}) \mathbf{k} \otimes \mathbf{l}]+\frac{\gamma-1}{2}(\mathbf{k}+\mathbf{l})|\mathbf{k}||\mathbf{l}|
\end{array}\right) \\
& \quad \times \frac{\mathrm{e}^{i(\mathbf{k}+\mathbf{l}) \cdot X}}{2\left|\mathbb{T}^{N}\right||\mathbf{k}||\mathbf{l}|} \mathrm{e}^{i(\alpha s g(\mathbf{k})|\mathbf{k}|+\beta s g(\mathbf{l})|\mathbf{l}|) s} .
\end{aligned}
$$

Projection on $V_{\mathbf{m}}^{\gamma}$ and on divergence-free vector fields, the function written above can be rewritten as follows $(F=P F+Q F)$

$$
\begin{gathered}
Q F(s)=\sum_{\substack{\mathbf{k}+\mathbf{l}=\mathbf{m} \\
\alpha, \beta, \gamma= \pm}} a_{\mathbf{k}}^{\alpha} a_{\mathbf{l}}^{\beta} \chi_{\mathbf{k} \mathbf{l}}^{\alpha \beta \gamma} V_{m}^{\gamma} \exp (i(\alpha s g(\mathbf{k})|\mathbf{k}|+\beta s g(\mathbf{l})|\mathbf{l}|-\gamma s g(\mathbf{m})|\mathbf{m}|) s), \\
P F(s)=\sum_{\substack{\mathbf{k}+\mathbf{l}=\mathbf{m} \\
\alpha, \beta= \pm}} a_{\mathbf{k}}^{\alpha} a_{\mathbf{l}}^{\beta} U_{\mathbf{k} \mathbf{m}}^{\alpha \beta} \exp (i(\alpha s g(\mathbf{k})|\mathbf{k}|+\beta s g(\mathbf{l})|\mathbf{l}|) s),
\end{gathered}
$$


where $\chi_{\mathbf{k l m}}^{\alpha \beta \gamma}$ is a constant and $U_{\mathbf{k l m}}^{\alpha \beta}=\left(0, u_{\mathbf{k l m}}^{\alpha \beta}\right)$ is a vector such that $\mathbf{m} \cdot u_{\mathbf{k l m}}^{\alpha \beta}=0$. Moreover, it is easy to see that $F$ is almost-periodic in $s$ with periods of the form $\alpha s g(\mathbf{k})|\mathbf{k}|+\beta s g(\mathbf{l})|\mathbf{I}|-\gamma \operatorname{sg}(\mathbf{k}+\mathbf{l})|\mathbf{k}+\mathbf{l}|$ and $\alpha s g(\mathbf{k})|\mathbf{k}|+\beta s g(\mathbf{l})|\mathbf{I}|$. Hence, to obtain the limit term in (30), we have to compute the mean value of (52). This is the same as looking at the resonant terms, namely terms which do not depend on $s$. The resonance condition between $((\mathbf{k}, \alpha),(\mathbf{l}, \beta),(\mathbf{m}, \gamma))$, namely $\left(V_{\mathbf{k}}^{\alpha}, V_{\mathbf{l}}^{\beta}, V_{\mathbf{m}}^{\gamma}\right)$ is

$$
\left\{\begin{array}{l}
\mathbf{k}+\mathbf{l}=\mathbf{m}, \\
\alpha \operatorname{sg}(\mathbf{k})|\mathbf{k}|+\beta s g(\mathbf{l})|\mathbf{l}|=\gamma \operatorname{sg}(\mathbf{m})|\mathbf{m}| .
\end{array}\right.
$$

Hence, $2 \mathbf{k} . \mathbf{l}=2 \alpha \operatorname{sg}(\mathbf{k}) \beta \operatorname{sg}(\mathbf{l})|\mathbf{k}||\mathbf{I}|$, which means that $\mathbf{k}$ is parallel to $\mathbf{l}$. Rewriting this product again and using that $\mathbf{k}$ is parallel to $\mathbf{l}$, we deduce that $\mathbf{k . l}=\operatorname{sg}(\mathbf{k}) \operatorname{sg}(\mathbf{l})|\mathbf{k}||\mathbf{I}|$. This yields that we have $\alpha=\beta$ and then we can see easily that (55) is equivalent to

$$
\left\{\begin{array}{l}
\mathbf{k}+\mathbf{l}=\mathbf{m}, \quad s g(\mathbf{k})|\mathbf{k}|+s g(\mathbf{I})|\mathbf{l}|=s g(\mathbf{m})|\mathbf{m}|, \\
\alpha=\beta=\gamma .
\end{array}\right.
$$

More precisely, we can only get resonances between the triplet $\left(V_{\mathbf{k}}^{+}, V_{\mathbf{l}}^{+}, V_{\mathbf{m}}^{+}\right)$and $\left(V_{\mathbf{k}}^{-}, V_{\mathbf{1}}^{-}, V_{\mathbf{m}}^{-}\right)$separately. This was the reason why we have introduced the notation $s g(\mathbf{k})$. On the other hand, the possible contribution on the divergence-free part requires the following resonance condition $\alpha \operatorname{sg}(\mathbf{k})|\mathbf{k}|+\beta \operatorname{si}(\mathbf{l})|\mathbf{I}|=0$ and hence $|\mathbf{k}|=|\mathbf{I}|$. Next, using the symmetry between $\mathbf{k}$ and $\mathbf{l}$, we get

$$
\begin{aligned}
u_{\mathbf{k l m}}^{\alpha \beta}+u_{\mathbf{l k m}}^{\beta \alpha}= & P\left[\alpha \left(\beta \operatorname{sg}(\mathbf{k}) \operatorname{sg}(\mathbf{l})((\mathbf{k}+\mathbf{l}) \cdot \mathbf{l} \mathbf{k}+(\mathbf{k}+\mathbf{l}) \cdot \mathbf{k} \mathbf{l}) \mathrm{e}^{i(\mathbf{k}+\mathbf{l}) X}\right.\right. \\
& \left.+\frac{\gamma-1}{2}(\mathbf{k}+\mathbf{l})|\mathbf{k}||\mathbf{l}| \mathrm{e}^{i(\mathbf{k}+\mathbf{l}) X}\right]=0 .
\end{aligned}
$$

The above relationship has already been used by Lions and the author in [12] to show the weak convergence of the compressible Navier-Stokes equations towards the incompressible Navier-Stokes equations. It means in some sense that the acoustic waves do not perturb the incompressible flow.

Finally, we deduce that

$$
\mathcal{Q}_{2}(V, V)=\sum_{\substack{\mathbf{k}+\mathbf{l}=\mathbf{m}, \alpha= \pm s g(\mathbf{k})|\mathbf{k}|+s g(\mathbf{l})|\mathbf{l}|=s g(\mathbf{m})|\mathbf{m}|}} a_{\mathbf{k}}^{\alpha} a_{\mathbf{l}}^{\alpha} \chi_{\mathbf{k} \mathbf{l} \mathbf{m}}^{\alpha} V_{\mathbf{m}}^{\alpha},
$$

where $\chi$ is symmetric in $\mathbf{k}$ and $\mathbf{l}$. It only remains to compute $\chi$. By projecting (30) on $V_{\mathbf{m}}^{\alpha}$, we get

$$
\begin{aligned}
\chi_{\mathbf{k l m}}^{\alpha} & =\frac{(-\alpha s g(\mathbf{m}) \mathbf{m})}{{\sqrt{2 \mid \mathbb{T}^{N}}}^{3}|\mathbf{k}||\mathbf{I}||\mathbf{m}|} \cdot\left(i \operatorname{sg}(\mathbf{k}) \operatorname{sg}(\mathbf{l}) \mathbf{m} \cdot \mathbf{k} \mathbf{l}+\frac{\gamma-1}{2} i|\mathbf{k}||\mathbf{I}| \mathbf{m}\right) \\
& =-i \alpha \frac{(\gamma+1) \operatorname{sg}(\mathbf{m})|\mathbf{m}|}{4 \sqrt{2\left|\mathbb{T}^{N}\right|}} .
\end{aligned}
$$

Inorder to understand more the structure of $\mathcal{Q}_{2}$, we introduce the set $\mathcal{P}$ of prime vectors $\mathbf{p}$, where $\mathbf{p} \in \mathbb{Z}^{N}$ is such that the $N$ components of $\mathbf{p}$ are prime in their set. 
This is equivalent to saying that there does not exist any couple $(n, \mathbf{q}) \in \mathbb{N} \times \mathbb{Z}^{N}$ such that $\mathbf{p}=n \mathbf{q}$ and $n \geqslant 2$. Then, we define

$$
W_{\mathbf{p}}(X)=\sum_{k \in \mathbb{Z}, \mathbf{k}=k \mathbf{p}} a_{\mathbf{k}}^{s g(\mathbf{p})} V_{\mathbf{k}}^{s g(\mathbf{p})}(X) .
$$

We can associate to the above vector value function the following real value function

$$
w_{\mathbf{p}}(z)=-\frac{\gamma+1}{4 \sqrt{2\left|\mathbb{T}^{N \mid}\right|}} \sum_{k \in \mathbb{Z}, \mathbf{k}=k \mathbf{p}} a_{\mathbf{k}}^{s g(\mathbf{p})} \mathrm{e}^{i k z} .
$$

We notice that both $W_{\mathbf{p}}$ and $w_{\mathbf{p}}$ are real. Indeed, this is a consequence of the fact that $V$ is real. Moreover, we remark that $\operatorname{sg}(\mathbf{p}) \operatorname{sg}(\mathbf{k})|\mathbf{k}|=|\mathbf{p}| k$ and that for all $s \in \mathbb{R}$, we have

$$
2 \pi \frac{\gamma+1}{4 \sqrt{2\left|\mathbb{T}^{N}\right|}}\left\|W_{\mathbf{p}}\right\|_{H^{s}\left(\mathbb{T}^{N}\right)}=|\mathbf{p}|^{s}\left\|w_{\mathbf{p}}\right\|_{H^{s}(\mathbb{T})} .
$$

The following proposition is very easy.

Proposition 4.1. - For all $\mathbf{p}, \mathbf{q} \in \mathcal{P}, \mathbf{p} \neq \mathbf{q}$, we have $\mathcal{Q}_{2}\left(W_{\mathbf{p}}, W_{\mathbf{q}}\right)=0$ and the following differential equations are equivalent

$$
\begin{gathered}
\partial_{t} V+\mathcal{Q}_{2}(V, V)-v \Delta V=0, \\
\forall \mathbf{p} \in \mathcal{P}, \quad \partial_{t} w_{\mathbf{p}}+|\mathbf{p}| \partial_{z} w_{\mathbf{p}}^{2}-v|\mathbf{p}|^{2} \partial_{z}^{2} w_{\mathbf{p}}=0 .
\end{gathered}
$$

This shows that we have to solve an infinite collection of viscous Burgers equations. However, our initial equation is even more complicated and we will see that this collection of viscous Burgers equations is coupled by a coupling coming from the term $Q_{1}$.

\subsection{Computation of $\mathcal{Q}_{1}$}

We recall that if $u$ is divergence-free it may be written as

$$
u=\sum_{\mathbf{k}} U_{\mathbf{k}} \mathrm{e}^{i \mathbf{k} \cdot X},
$$

where $\mathbf{k} \cdot U_{\mathbf{k}}=0$, for all $\mathbf{k}$. Then, using the fact that $u$ is real, we deduce that $U_{\mathbf{k}}=\overline{U_{-\mathbf{k}}}$, for all $\mathbf{k}$. Hence, the term inside the integral in the right hand side of (29) is given by

$$
\mathcal{L}(-s) \sum_{\substack{\mathbf{k}, \mathbf{l} \\
\alpha= \pm}} a_{\mathbf{k}}^{\alpha} \frac{1}{\sqrt{2\left|\mathbb{T}^{N}\right||\mathbf{k}|}} i\left(\begin{array}{c}
0 \\
\alpha s g(\mathbf{k})(\mathbf{k}+\mathbf{l}) \cdot\left[\mathbf{k} \otimes U_{\mathbf{l}}+U_{\mathbf{l}} \otimes k\right]
\end{array}\right) \mathrm{e}^{i(\mathbf{k}+\mathbf{l}) \cdot X} \mathrm{e}^{i \alpha s g(\mathbf{k})|\mathbf{k}| s} .
$$

Now, we have to look at the resonant terms as we did in the preceding section for $\mathcal{Q}_{2}$. The resonance condition between $((\mathbf{k}, \alpha), \mathbf{l},(\mathbf{m}, \gamma))$, namely $\left(V_{\mathbf{k}}^{\alpha}, U_{\mathbf{l}}, V_{\mathbf{m}}^{\gamma}\right)$ is

$$
\left\{\begin{array}{l}
\mathbf{k}+\mathbf{l}=\mathbf{m}, \\
\alpha s g(\mathbf{k})|\mathbf{k}|=\delta s g(\mathbf{m})|\mathbf{m}| .
\end{array}\right.
$$


We just notice that if $\left(V_{\mathbf{k}}^{\alpha}, U_{\mathbf{l}}, V_{\mathbf{m}}^{\delta}\right)$ is a resonant triplet than it is also true for $\left(V_{-\mathbf{m}}^{\delta}, U_{\mathbf{l}}, V_{-\mathbf{k}}^{\alpha}\right)$. We will see that this yields the conservation of energy. Of course, we also get other resonances for instance for $\left(V_{-\mathbf{k}}^{\alpha}, U_{-\mathbf{l}}, V_{-\mathbf{m}}^{\delta}\right) \ldots$ Next, we get

$$
\mathcal{Q}_{1}(u, V)=\sum_{\substack{\mathbf{k}+\mathbf{l}=\mathbf{m} \\ \alpha s g(\mathbf{k})=\delta s g(\mathbf{m}) \\|\mathbf{k}|=|\mathbf{m}|}} a_{\mathbf{k}}^{\alpha} \sigma\left(\mathbf{k}, U_{\mathbf{l}}\right) V_{\mathbf{m}}^{\delta},
$$

where

$$
\begin{aligned}
\sigma\left(\mathbf{k}, U_{\mathbf{l}}\right) & =\frac{(-\delta s g(\mathbf{m}) \mathbf{m})}{2|\mathbf{k}||\mathbf{m}|} \cdot\left(i \alpha s g(\mathbf{k})\left[\mathbf{m} \cdot \mathbf{k} U_{\mathbf{l}}+\mathbf{k} \cdot U_{\mathbf{l}} \mathbf{k}\right]\right) \\
& =-i \frac{\mathbf{m} \cdot \mathbf{k}}{|\mathbf{k}||\mathbf{m}|} U_{\mathbf{l}} \cdot \mathbf{k} .
\end{aligned}
$$

Moreover, using the fact that $\mathbf{l} . U_{\mathrm{l}}=0$, we get that $\sigma\left(-\mathbf{m}, U_{\mathrm{l}}\right)=-\sigma\left(\mathbf{k}, U_{\mathrm{l}}\right)$. It must be noticed that while $\mathcal{Q}_{2}(V, V)$ is formed by resonances between modes oscillating in the same direction, $\mathcal{Q}_{1}(u, V)$ is formed by resonances between modes oscillating with the same frequency. This induces some coupling in the limit equation (32) which can be seen as a an infinite collection of coupled viscous Burgers equations. However, we will see that for suitable choices of the periods of the domain this coupling is low. Next, we introduce the following set, that we call the set of trivial resonances

$$
\mathcal{A}_{\mathbf{p}}=\left\{\mathbf{q} \in \mathcal{P}, \forall i, \mathbf{q}_{i}= \pm \mathbf{p}_{i}\right\}
$$

PROPOSITION 4.2. - For almost all choices of $\left(a_{1}, \ldots, a_{N}\right) \in \mathbb{R}^{+N}, \mathcal{Q}_{1}(u, V)$ reduces to trivial resonances, namely

$$
\mathcal{Q}_{1}(u, V)=\sum_{\mathbf{k} \in \mathcal{A}_{\mathbf{m}}, \alpha s g(\mathbf{k})=\delta s g(\mathbf{m})} a_{\mathbf{k}}^{\alpha} \sigma^{\alpha}\left(\mathbf{k}, U_{\mathbf{m}-\mathbf{k}}\right) V_{\mathbf{m}}^{\delta}
$$

More precisely if $\frac{1}{a_{1}^{2}}, \ldots, \frac{1}{a_{N}^{2}}$ are $\mathbb{Q}$ independent then the above conclusion holds.

The proof of this proposition is very easy. In fact, if $\frac{1}{a_{1}^{2}}, \ldots, \frac{1}{a_{N}^{2}}$ are $\mathbb{Q}$ independent, then the equation

$$
\sum_{i=1}^{N} \frac{\mathbf{k}_{i}^{\prime 2}}{a_{i}^{2}}=\sum_{i=1}^{N} \frac{\mathbf{m}_{i}^{\prime 2}}{a_{i}^{2}}
$$

has only trivial solutions, namely $\mathbf{k}_{i}^{\prime 2}=\mathbf{m}_{i}^{\prime 2}$, which means that $\mathbf{k} \in \mathcal{A}_{\mathbf{m}}$. Moreover in this case the resonance also holds for the prime represent of $(\mathbf{k}, \alpha)$ and $(\mathbf{m}, \delta)$, namely $\mathbf{q}$ and $\mathbf{p}$ such that $s g(\mathbf{q})=\alpha, \mathbf{k}=k \mathbf{q}$ and $s g(\mathbf{p})=\delta, \mathbf{m}=m \mathbf{p}$, since $k=m$ in this case. However, in the general case, the resonances couple all the different modes. 
To be able to use the notations $W_{\mathbf{p}}$ to analyze the limit system, we define $C_{\mathbf{p}}\left(u, W_{\mathbf{q}}\right)$ which is the contribution of $W_{\mathbf{q}}$ on $W_{\mathbf{p}}$

$$
C_{\mathbf{p}}\left(u, W_{\mathbf{q}}\right)=\sum_{\substack{\mathbf{k}+\mathbf{l}=\mathbf{m} \\ \mathbf{k}=\mathbf{k}, \mathbf{m}=m \mathbf{p} \\ m|\mathbf{p}|=k|\mathbf{q}| a_{\mathbf{k}}^{s g(\mathbf{q})}}} \sigma\left(\mathbf{k}, U_{\mathbf{l}}\right) V_{\mathbf{m}}^{s g(\mathbf{p})}
$$

Indeed, using that $\mathbf{k}=k \mathbf{q}, \mathbf{m}=m \mathbf{p}$, the second equation in (68) yields

$$
\operatorname{sg}(\mathbf{q}) \operatorname{sg}(\mathbf{k})|\mathbf{k}|=\operatorname{sg}(\mathbf{p}) \operatorname{sg}(\mathbf{m})|\mathbf{m}|,
$$

from which we deduce the condition $m|\mathbf{p}|=k|\mathbf{q}|$. We also introduce the corresponding notation for $w_{\mathbf{p}}$

$$
c_{\mathbf{p}}\left(u, w_{\mathbf{q}}\right)=\sum_{\mathbf{k}+\mathbf{l}=\mathbf{m}, \mathbf{k}=k \mathbf{q}, \mathbf{m}=m \mathbf{p}, m|\mathbf{p}|=k|\mathbf{q}|} a_{\mathbf{k}}^{s g(\mathbf{q})} \sigma\left(\mathbf{k}, U_{\mathbf{l}}\right) \mathrm{e}^{i m z} .
$$

With these notations, we can see that

PROPOSITION 4.3. - Solving the system (32) for $V=\sum_{\mathbf{p} \in \mathcal{P}} W_{\mathbf{p}}$ is equivalent to solving the system (ICVB) of infinite coupled viscous Burgers equations

$$
(I C V B) \quad \forall \mathbf{p} \in \mathcal{P}, \quad \partial_{t} w_{\mathbf{p}}+|\mathbf{p}| \partial_{z} w_{\mathbf{p}}^{2}-v|\mathbf{p}|^{2} \partial_{z}^{2} w_{\mathbf{p}}+\sum_{\mathbf{q} \in \mathcal{P}} c_{\mathbf{p}}\left(u, w_{\mathbf{q}}\right)=0 .
$$

We also notice that, when $v=0$, the system reduces to an infinite coupled Burgers equations

$$
\text { (ICB) } \quad \forall \mathbf{p} \in \mathcal{P}, \quad \partial_{t} w_{\mathbf{p}}+|\mathbf{p}| \partial_{z} w_{\mathbf{p}}^{2}+\sum_{\mathbf{q} \in \mathcal{P}} c_{\mathbf{p}}\left(u, w_{\mathbf{q}}\right)=0 .
$$

The proof of the existence of solutions will rely on the energy estimates. Therefore, we begin by proving Proposition 3.3, which is the essence of the conservation of the energy.

\subsection{Proof of Proposition 3.3}

Let us start by proving the first part of Eq. (33). It is easy to see that if $\left(V_{\mathbf{k}}^{\alpha}, U_{\mathbf{l}}, V_{\mathbf{m}}^{\delta}\right)$ is resonant then it is also true for $\left(V_{-\mathbf{m}}^{\delta}, U_{\mathbf{l}}, V_{-\mathbf{k}}^{\alpha}\right)$. Moreover using that $V$ is real, we deduce that $a_{-\mathbf{k}}^{+}=\overline{a_{\mathbf{k}}^{+}}$and hence the following computations are obvious

$$
\begin{aligned}
& \int \mathcal{Q}_{1}(u, V) V=\sum_{\substack{\mathbf{k}+\mathbf{l}=\mathbf{m}, \alpha s g(\mathbf{k})=\delta s g(\mathbf{m}) \\
|\mathbf{k}|=|\mathbf{m}|}} a_{\mathbf{k}}^{\alpha} \sigma\left(\mathbf{k}, U_{\mathbf{l}}\right) a_{\mathbf{m}}^{\alpha} \\
& =\frac{1}{2} \sum_{\mathbf{k}+\mathbf{l}=\mathbf{m}, \underset{\alpha s g(\mathbf{k})=\delta s g(\mathbf{m})}{|\mathbf{k}|=|\mathbf{m}|}}-i \frac{\mathbf{m} \cdot \mathbf{k}}{|\mathbf{k}||\mathbf{m}|}\left(U_{\mathbf{l}} \cdot \mathbf{k} a_{\mathbf{k}}^{\alpha} a_{-\mathbf{m}}^{\alpha}-U_{\mathbf{l}} \cdot \mathbf{m} a_{\mathbf{k}}^{\alpha} a_{-\mathbf{m}}^{\alpha}\right) \\
& =0 \text {. }
\end{aligned}
$$


For the second part of Eq. (33), we just remark that if $\left(V_{\mathbf{k}}^{\alpha}, V_{\mathbf{l}}^{\alpha}, V_{\mathbf{m}}^{\alpha}\right)$ is a resonant triplet, it is also true for $\left(V_{\mathbf{k}}^{\alpha}, V_{-\mathbf{m}}^{\alpha}, V_{-\mathbf{l}}^{\alpha}\right)$ and $\left(V_{-\mathbf{m}}^{\alpha}, V_{\mathbf{l}}^{\alpha}, V_{-\mathbf{k}}^{\alpha}\right)$. Hence

$$
\begin{aligned}
\int \mathcal{Q}_{2}(V, V) V= & \sum_{\mathbf{k}+\mathbf{l}=\mathbf{m}, \alpha= \pm} a_{\mathbf{k}}^{\alpha} a_{\mathbf{l}}^{\alpha} \chi_{\mathbf{k} \mathbf{l} \mathbf{m}}^{\alpha} a_{-\mathbf{m}}^{\alpha} \\
= & \frac{1}{3} \sum_{\mathbf{k}+\mathbf{l}=\mathbf{m}, \alpha= \pm}-i \alpha \frac{(\gamma+1)}{4 \sqrt{2\left|\mathbb{T}^{N}\right|}}\left(\operatorname{sg}(\mathbf{m})|\mathbf{m}| a_{\mathbf{k}}^{\alpha} a_{\mathbf{l}}^{\alpha} a_{-\mathbf{m}}^{\alpha}\right. \\
& \left.+\operatorname{sg}(-\mathbf{l})|\mathbf{l}| a_{\mathbf{k}}^{\alpha} a_{-\mathbf{m}}^{\alpha} a_{\mathbf{l}}^{\alpha}+s g(-\mathbf{k})|\mathbf{k}| a_{\mathbf{l}}^{\alpha} a_{-\mathbf{m}}^{\alpha} a_{\mathbf{k}}^{\alpha}\right) \\
= & 0 .
\end{aligned}
$$

\subsection{Existence of global solutions for the coupled system (ICVB)}

In this subsection, we give a sketch of proof of existence and uniqueness of solutions to $(I C V B)$ and hence for (32). Let $u \in L^{\infty}\left(H^{s}\right)$, with $s \geqslant N / 2+1$ be a given function (the regularity of $u$ can be weakened but $u \in L^{\infty}\left(H^{s}\right)$ is the regularity we get from the fact that $u$ is a solution of the Euler system) and $V_{0}=\sum_{\mathbf{p}} W_{\mathbf{p}}^{0} \in H^{s-1}$, we also define $w_{\mathbf{p}}^{0}$ as above. Then, the following theorem holds

THEOREM 4.4. - There exists a unique global strong solution for (ICVB), with

$$
\forall \mathbf{p} \in \mathcal{P}, \quad w_{\mathbf{p}} \in L^{\infty}\left(H^{s-1}(\mathbb{T})\right) \cap L^{2}\left(H^{s}(\mathbb{T})\right)
$$

And hence, we obtain a unique strong solution for (32), with $V=\sum_{\mathbf{p}} W_{\mathbf{p}} \in$ $L^{\infty}\left(H^{s-1}\left(\mathbb{T}^{N}\right)\right) \cap L^{2}\left(H^{s}\left(\mathbb{T}^{N}\right)\right)$.

We are just going to give the a priori bounds we can derive for this system. The existence result is then easily deduced by solving some approximated systems. For instance, we can solve $\left(I C V B_{M}\right)$ for all $M \in \mathbb{N}$, defined by $\mathcal{P}_{M}=\{\mathbf{p} \in \mathcal{P},|\mathbf{p}| \leqslant M\}$ and

$$
\left(I C V B_{M}\right) \quad \forall \mathbf{p} \in \mathcal{P}_{M}, \quad \partial_{t} w_{\mathbf{p}}+|\mathbf{p}| \partial_{z} w_{\mathbf{p}}^{2}-v|\mathbf{p}|^{2} \partial_{z}^{2} w_{\mathbf{p}}+\sum_{\mathbf{q} \in \mathcal{P}_{M}} c_{\mathbf{p}}\left(u, w_{\mathbf{q}}\right)=0
$$

Of course all the a priori estimates we are going to show for (ICVB) can be easily extended to $\left(I C V B_{M}\right)$. Then, we have just to take the limit $M \rightarrow \infty$ and use a compactness method to pass to the limit. To solve $\left(I C V B_{M}\right)$ for fixed $M$, we can use any classical type of regularization. For instance, one can use a Galerkin approximation method.

Now, we turn to the proof of the a priori bounds. If Proposition 3.3 is sufficient to get $L^{2}$ energy estimates, we need the following Proposition to get higher order estimates.

Proposition 4.5. - For all $\mathbf{p}, \mathbf{q}, \in \mathcal{P}$ and all $j \in N$, we have

$$
\int_{\mathbb{T}} \partial_{z}^{j} c_{\mathbf{p}}\left(u, w_{\mathbf{q}}\right)|\mathbf{p}|^{2 j} \partial_{z}^{j} w_{\mathbf{p}}+\int_{\mathbb{T}} \partial_{z}^{j} c_{\mathbf{q}}\left(u, w_{\mathbf{p}}\right)|\mathbf{q}|^{2 j} \partial_{z}^{j} w_{\mathbf{q}}=0
$$


Proof. - It relies upon the following observations. Since for all resonant triplet $\left(V_{\mathbf{k}}^{\alpha}, U_{\mathbf{l}}, V_{\mathbf{m}}^{\delta}\right)$ (with $s g(\mathbf{q})=\alpha, \mathbf{k}=k \mathbf{q}$ and $\left.s g(\mathbf{p})=\delta, \mathbf{m}=m \mathbf{p}\right)$, we know that $\left(V_{-\mathbf{m}}^{\delta}, U_{\mathbf{l}}, V_{-\mathbf{k}}^{\alpha}\right)$ is also resonant and $\sigma\left(-\mathbf{m}, U_{\mathbf{l}}\right)=-\sigma\left(\mathbf{k}, U_{\mathbf{l}}\right)$, we obtain

$$
a_{-\mathbf{m}}^{\delta} \sigma\left(-\mathbf{m}, U_{\mathbf{l}}\right)|\mathbf{k}|^{2 j} a_{\mathbf{k}}^{\alpha}+a_{\mathbf{k}}^{\alpha} \sigma\left(\mathbf{k}, U_{\mathbf{l}}\right)|\mathbf{m}|^{2 j} a_{-\mathbf{m}}^{\delta}=0 .
$$

Here, we have also used that $|\mathbf{m}|^{2 j}=|m|^{2 j}|\mathbf{p}|^{2 j}=|\mathbf{k}|^{2 j}=|k|^{2 j}|\mathbf{q}|^{2 j}$. Then, we conclude easily.

Using Proposition 3.3, we get the following $L^{2}$ energy estimate for all $t$

$$
\frac{1}{2} \sum_{\mathbf{p} \in \mathcal{P}}\left\|w_{\mathbf{p}}\right\|_{L^{2}}^{2}(t)+v \int_{0}^{t} \sum_{\mathbf{p} \in \mathcal{P}}|p|^{2}\left\|\partial_{z} w_{\mathbf{p}}\right\|_{L^{2}}^{2} \leqslant \frac{1}{2} \sum_{\mathbf{p} \in \mathcal{P}}\left\|w_{\mathbf{p}}\right\|_{L^{2}}^{2}(0)=C\left\|V_{0}\right\|_{L^{2}}^{2} .
$$

Next, for all $j \in N$ and all $\mathbf{p} \in \mathcal{P}$, we have

$$
\begin{aligned}
& \frac{1}{2} \partial_{t}\left\|\partial^{j} w_{\mathbf{p}}\right\|_{L^{2}}^{2}+v|\mathbf{p}|^{2}\left\|\partial^{j+1} w_{\mathbf{p}}\right\|_{L^{2}}^{2}+|\mathbf{p}| \int_{\mathbb{T}} \partial^{j+1}\left(w_{\mathbf{p}}^{2}\right) \partial^{j} w_{\mathbf{p}} \\
& \quad+\int_{\mathbb{T}} \sum_{\mathbf{q}} \partial^{j} c_{\mathbf{p}}\left(u, w_{\mathbf{q}}\right) \partial^{j} w_{\mathbf{p}}=0 .
\end{aligned}
$$

To estimate $\int_{\mathbb{T}} \partial^{j+1}\left(w_{\mathbf{p}}^{2}\right) \partial^{j} w_{\mathbf{p}}$, we need the following

PROPOSITION 4.6. - Using interpolation inequality, we get

$$
\int_{\mathbb{T}} \partial^{j+1}\left(w_{\mathbf{p}}^{2}\right) \partial^{j} w_{\mathbf{p}} \leqslant C\left\|w_{\mathbf{p}}\right\|_{L^{\infty}}\left\|\partial^{j} w_{\mathbf{p}}\right\|_{L^{2}}\left\|\partial^{j+1} w_{\mathbf{p}}\right\|_{L^{2}} .
$$

Proof. - Indeed, by Hölder inequality, we find

$$
\begin{aligned}
\int_{\mathbb{T}} \partial^{j+1}\left(w_{\mathbf{p}}^{2}\right) \partial^{j} w_{\mathbf{p}} \leqslant & \sum_{\substack{s+r=j+1 \\
j \geqslant r, s \geqslant 1}}\left(\begin{array}{c}
j+1 \\
r
\end{array}\right)\left\|\partial^{r} w_{\mathbf{p}}\right\|_{L^{4}}\left\|\partial^{s} w_{\mathbf{p}}\right\|_{L^{4}}\left\|\partial^{j} w_{\mathbf{p}}\right\|_{L^{2}} \\
& +2\left\|w_{\mathbf{p}}\right\|_{L^{\infty}}\left\|\partial^{j+1} w_{\mathbf{p}}\right\|_{L^{2}}\left\|\partial^{j} w_{\mathbf{p}}\right\|_{L^{2}} .
\end{aligned}
$$

Then, using Gagliardo-Nirenberg's inequality, we deduce

$$
\left\|\partial^{r} w_{\mathbf{p}}\right\|_{L^{4}} \leqslant C\left\|w_{\mathbf{p}}\right\|_{L^{\infty}}^{1-\theta}\left\|\partial^{j+1} w_{\mathbf{p}}\right\|_{L^{2}}^{\theta},
$$

where $\frac{1}{4}-s=\theta\left(\frac{1}{2}-(j+1)\right)$. And, since $r+s=j+1$, we also have

$$
\left\|\partial^{s} w_{\mathbf{p}}\right\|_{L^{4}} \leqslant C\left\|w_{\mathbf{p}}\right\|_{L^{\infty}}^{\theta}\left\|\partial^{j+1} w_{\mathbf{p}}\right\|_{L^{2}}^{1-\theta}
$$

and the proposition is proved.

Then, multiplying (85) by $|\mathbf{p}|^{2 j}$ and summing up in $\mathbf{p}$, we get 


$$
\begin{aligned}
& \frac{1}{2} \partial_{t}\|V\|_{H^{j}}^{2}+v\|V\|_{H^{j+1}}^{2} \leqslant \sum_{\mathbf{p}}\left\|w_{\mathbf{p}}\right\|_{L^{\infty}}|\mathbf{p}|^{2 j+1}\left\|\partial^{j} w_{\mathbf{p}}\right\|_{L^{2}}\left\|\partial^{j+1} w_{\mathbf{p}}\right\|_{L^{2}} \\
& \leqslant \frac{C}{v} \sum_{\mathbf{p}}\left\|w_{\mathbf{p}}\right\|_{L^{\infty}}|\mathbf{p}|^{2 j}\left\|\partial^{j} w_{\mathbf{p}}\right\|_{L^{2}}^{2}+\frac{v}{2} \sum_{\mathbf{p}}|\mathbf{p}|^{2 j+2}\left\|\partial^{j} w_{\mathbf{p}}\right\|_{L^{2}}^{2},
\end{aligned}
$$

where we have used Cauchy-Schwarz inequality. This yields for all $t$

$$
\partial_{t}\|V\|_{H^{j}}^{2}+v\|V\|_{H^{j+1}}^{2} \leqslant \frac{C}{v}\left(\sup _{p}\left\|w_{\mathbf{p}}\right\|_{L^{\infty}}^{2}\right)\|V\|_{H^{j}}^{2} \leqslant \frac{C}{v}\left(\sum_{p}\left\|w_{\mathbf{p}}\right\|_{L^{\infty}}^{2}\right)\|V\|_{H^{j}}^{2}
$$

Then, by Gagliardo-Nirenberg's inequality, we have for all $t$,

$$
\sum_{\mathbf{p}}\left\|w_{\mathbf{p}}\right\|_{L^{\infty}}^{2} \leqslant \sum_{\mathbf{p}}\left\|w_{\mathbf{p}}\right\|_{L^{2}}\left\|\partial_{z} w_{\mathbf{p}}\right\|_{L^{2}} \leqslant\left(\sum_{\mathbf{p}}\left\|w_{\mathbf{p}}\right\|_{L^{2}}^{2}\right)^{1 / 2}\left(\sum_{\mathbf{p}}\left\|\partial_{z} w_{\mathbf{p}}\right\|_{L^{2}}^{2}\right)^{1 / 2} .
$$

Integrating in time and using again Cauchy-Schwarz' inequality, we obtain

$$
\int_{0}^{T} \sum_{\mathbf{p}}\left|w_{\mathbf{p}}\right|_{L^{\infty}}^{2} \leqslant \frac{C \sqrt{T}}{\sqrt{v}}
$$

Finally, by Grönwall inequality, we find for all $t$

$$
\|V\|_{H^{j}}^{2}(t)+v \int_{0}^{t}\|V\|_{H^{j+1}}^{2} \leqslant C\left\|V_{0}\right\|_{H^{j}}^{2} \exp \left(\frac{C \sqrt{t}}{v^{3 / 2}}\right)
$$

We want to remark, that it is possible to get an estimate independent of $t$, by noticing that since $w_{\mathbf{p}}$ has a zero average then $\left\|w_{\mathbf{p}}\right\|_{L^{\infty}} \leqslant\left\|\partial_{z} w_{\mathbf{p}}\right\|_{L^{2}}$. This yields the following estimate

$$
\|V\|_{H^{j}}^{2}(t)+v \int_{0}^{t}\|V\|_{H^{j+1}}^{2} \leqslant C\left\|V_{0}\right\|_{H^{j}}^{2} \exp \left(\frac{C}{v^{2}}\right) .
$$

The argument, we presented here uses the fact that $j \in \mathbb{N}$. However, it can be easily adapted to the case where $s \in \mathbb{R}$, hence we get that

$$
\|V\|_{H^{s}}^{2}(t)+v \int_{0}^{t}\|V\|_{H^{s+1}}^{2} \leqslant C\left\|V_{0}\right\|_{H^{s}}^{2} \exp \left(\frac{C \sqrt{t}}{v^{3 / 2}}\right)
$$

and we conclude.

\subsection{Existence of local solutions for the coupled system (ICB)}

In this case the proof is the same, apart from the fact that we can no longer use the effect of the viscosity. Hence, inequality (86) should be replaced by

$$
\int_{\mathbb{T}} \partial^{j+1}\left(w_{\mathbf{p}}^{2}\right) \partial^{j} w_{\mathbf{p}} \leqslant\left\|\partial_{z} w_{\mathbf{p}}\right\|_{L^{\infty}}\left\|\partial^{j} w_{\mathbf{p}}\right\|_{L^{2}}^{2}
$$


Indeed, computing $\partial^{j+1}\left(w_{\mathbf{p}}^{2}\right)$, we have

$$
\partial^{j+1}\left(w_{\mathbf{p}}^{2}\right)=2 w_{\mathbf{p}} \partial^{j+1} w_{\mathbf{p}}+2(j+1) \partial w_{\mathbf{p}} \partial^{j} w_{\mathbf{p}}+\sum_{\substack{r+s=j+1 \\ j-1 \geqslant r, s \geqslant 2}} \partial^{r} w_{\mathbf{p}} \partial^{s} w_{\mathbf{p}} .
$$

The first term in the left hand side can be treated as follows

$$
\int_{\mathbb{T}} w_{\mathbf{p}} \partial^{j+1} w_{\mathbf{p}} \partial^{j} w_{\mathbf{p}}=\int_{\mathbb{T}} w_{\mathbf{p}} \partial\left(\partial^{j} w_{\mathbf{p}}\right)^{2}=-\int_{\mathbb{T}} \partial w_{\mathbf{p}}\left(\partial^{j} w_{\mathbf{p}}\right)^{2}
$$

For the others, we conclude as in the proof of Proposition 4.6.

Besides, inequality (90) must be replaced by

$$
\partial_{t}\|V\|_{H^{j}}^{2} \leqslant C\|V\|_{H^{j}}^{3}
$$

In fact, since $j>3 / 2$, we deduce that $\left\|\partial_{z} w_{\mathbf{p}}\right\|_{L^{\infty}} \leqslant\left\|\partial^{j} w_{\mathbf{p}}\right\|_{L^{2}}$. Finally, it is easy to see that (98) yields the local existence for $(I C B)$.

\section{Acknowledgements}

The author wishes to thank P.L. Lions for many discussions about this work.

\section{REFERENCES}

[1] Desjardins B., Grenier E., Low Mach number limit of compressible viscous flows in the whole space, Preprint.

[2] Desjardins B., Grenier E., Lions P.-L., Masmoudi N., Compressible incompressible limit with Dirichlet boundary condition, J. Math. Pures et Appl. 78 (5) (1999) 461-471.

[3] Gallagher I., A remark on smooth solutions of the weakly compressible periodic NavierStokes equations, Preprint, 1999.

[4] Grenier E., Oscillatory perturbations of the Navier-Stokes equations, J Math. Pures et Appl. 976 (6) (1997) 477-498.

[5] Hagstrom T., Lorenz J., All-time existence of classical solutions for slightly compressible flows, SIAM J. Math. Anal. 29 (3) (1998) 652-672.

[6] Klainerman S., Majda A., Singular limits of quasilinear hyperbolic systems with large parameters and the incompressible limit of compressible fluids, Comm. Pure Appl. Math. 34 (5) (1981) 481-524.

[7] Klainerman S., Majda A., Compressible and incompressible fluids, Comm. Pure Appl. Math. 35 (5) (1982) 629-651.

[8] Kreiss H.O., Lorentz J., Naughton M.J., Convergence of the solutions of the compressible to the solutions of the incompressible Navier-Stokes equations, Adv. in Appl. Math. 12 (2) (1991) 187-214.

[9] Lin C.K., On the incompressible limit of the compressible Navier-Stokes equations, Comm. Partial Differential Equations 20 (3-4) (1995) 677-707.

[10] Lions P.L., Mathematical Topics in Fluid Dynamics, Vol. 1: Incompressible Models, Oxford University Press, 1996. 
[11] Lions P.L., Mathematical Topics in Fluid Dynamics, Vol. 2: Compressible Models, Oxford University Press, 1998.

[12] Lions P.L., Masmoudi N., Incompressible limit for a viscous compressible fluid, J. Math. Pures Appl. 77 (1998) 585-627.

[13] Lions P.L., Masmoudi N., On a free boundary barotropic model, Annales de 1'IHP, Analyse Non Linaire 16 (1999) 373-410.

[14] Lions P.L., Masmoudi N., Une approche locale de le limite incompressibel, C. R. Acad. Sci. Paris Sr. I Math. 329 (5) (1999) 387-392.

[15] Masmoudi N., The Euler limit of the Navier-Stokes equations, and rotating fluids with boundary, Arch. Rational Mech. Anal. 142 (1998) 375-394.

[16] Masmoudi N., Ekman layers of rotating fluids, the case of general initial data, Comm. Pure Appl. Math. 53 (4) (2000) 432-483.

[17] Schochet S., Fast singular limits of hyperbolic PDEs, J. Differential Equations 114 (1994) $476-512$.

[18] Ukai S., The incompressible limit and the initial layer of the compressible Euler equation, J. Math. Kyoto Univ. 26 (2) (1986) 323-331. 